\title{
Luminescent Mononuclear and Binuclear Cyclometalated Palladium(II) Complexes of 6-Phenyl-2,2'-bipyridines: Spectroscopic and Structural Comparisons with Platinum(II) Analogues ${ }^{1,2}$
}

\author{
Siu-Wai Lai, Tsz-Chun Cheung, Michael C. W. Chan, Kung-Kai Cheung, \\ Shie-Ming Peng, ${ }^{\dagger}$ and Chi-Ming Che*
}

Department of Chemistry, The University of Hong Kong, Pokfulam Road, Hong Kong

Received September 13, 1999

\begin{abstract}
The mononuclear cyclometalated $\mathrm{Pd}(\mathrm{II})$ complexes $\left[\mathrm{Pd}\left(\mathrm{L}^{1}\right) \mathrm{X}\right]\left(\mathrm{HL}^{1}=6\right.$-phenyl-2,2'-bipyridine; $\mathrm{X}=\mathrm{Cl}, \mathbf{1 a} ; \mathrm{Br}$, 1b; I, 1c), $\left[\mathrm{Pd}\left(\mathrm{L}^{1}\right) \mathrm{PPh}_{3}\right]^{+}(\mathbf{1 d}),\left[\mathrm{Pd}\left(\mathrm{L}^{2-5}\right) \mathrm{Cl}\right]\left[\mathbf{2 a}-\mathbf{5 a}, \mathrm{HL}^{2-5}=4\right.$-(aryl)-6-phenyl-2,2'-bipyridine; aryl = phenyl (2), 4-chlorophenyl (3), 4-tolyl (4), 4-methoxyphenyl (5)] and the binuclear derivatives $\left[\mathrm{Pd}_{2}\left(\mathrm{~L}^{1-5}\right)_{2}(\mu \text {-dppm) }]^{2+}\right.$ $\left(\mathbf{1 e}-\mathbf{5 e}, \mathrm{dppm}=\right.$ bis $\left(\right.$ diphenylphosphino)methane) and $\left[\mathrm{Pd}_{2}\left(\mathrm{~L}^{1}\right)_{2}(\mu \text {-dppC })_{5}\right]^{2+},\left(\mathbf{1 f}, \mathrm{dppC}_{5}=1,5\right.$-bis(diphenylphosphino)pentane) were prepared. The crystal structures of $\mathbf{1 d}\left(\mathrm{ClO}_{4}\right), \mathbf{1 e}\left(\mathrm{ClO}_{4}\right)_{2} \cdot \mathrm{DMF}$, and $\mathbf{2 e}\left(\mathrm{ClO}_{4}\right)_{2}$ have been determined by X-ray crystallography. The magnitude of the $\mathrm{Pd}-\mathrm{Pd}$ distances in $\mathbf{1 e}$ and $\mathbf{2 e}(3.230(1)$ and 3.320(2) $\AA$, respectively) suggest minimal metal-metal interaction, although $\pi$-stacking of the aromatic ligands (interplanar separations 3.34 and $3.35 \AA$, respectively) is evident. All complexes display low-energy UV absorptions at $\lambda \sim$ $390 \mathrm{~nm}$, which are tentatively assigned to ${ }^{1}$ MLCT transitions; red shifts resulting from $\mathrm{Pd}-\mathrm{Pd}$ interactions in the binuclear species are not apparent. The complexes in this work are non-emissive at $298 \mathrm{~K}$, but the cationic derivatives exhibit intense luminescence at $77 \mathrm{~K}$. The structured emissions of $\mathbf{1 d}$ and $\mathbf{1 f}$ in $\mathrm{MeOH} / \mathrm{EtOH}$ glass $\left(\lambda_{\max } 467-586 \mathrm{~nm}\right)$ and all cationic species in the solid state $\left(\lambda_{\max } 493-578 \mathrm{~nm}\right)$ are assigned to intraligand excited states. Complexes $\mathbf{1 e}-\mathbf{5 e}$ display dual emissions in $\mathrm{MeOH} / \mathrm{EtOH}$ glass at $77 \mathrm{~K}$, and the broad structureless bands at $\lambda_{\max } 626-658 \mathrm{~nm}$ are attributed to $\pi-\pi$ excimeric IL transitions. A comparison between the photophysical properties of $\mathrm{Pd}(\mathrm{II})$ and $\mathrm{Pt}(\mathrm{II})$ congeners is presented.
\end{abstract}

\section{Introduction}

The photophysics and photoreactivity of coordinatively unsaturated platinum(II) complexes ${ }^{3}$ and their applications as luminescent sensors ${ }^{4}$ are areas of considerable interest in inorganic photochemistry. Rich and diverse low-energy excited states, including IL (intraligand: $\pi \rightarrow \pi^{*}$ ), MLCT (metal-toligand charge transfer), $\left[\mathrm{d} \sigma^{*} \rightarrow \mathrm{p} \sigma\right]$, and $\left[\mathrm{d} \sigma^{*} \rightarrow \pi^{*}\right]$ (or MMLCT, metal-metal-to-ligand charge transfer), have been observed. Relatively scant attention has been focused upon the luminescent characteristics of palladium(II) complexes. Nevertheless, such investigations have been dominated by cyclometalated derivatives, ${ }^{5-7}$ and reports comparing $\mathrm{Pd}(\mathrm{II})$ and $\mathrm{Pt}(\mathrm{II})$ analogues can yield information on electronic structures and

*Corresponding author. Fax: +852 2857 1586. Email: cmche@ hku.hk.

$\dagger$ Department of Chemistry, National Taiwan University, Taipei, Taiwan

(1) Lai, S. W.; Chan, M. C. W.; Cheung, T. C.; Peng, S. M.; Che, C. M. Inorg. Chem. 1999, 38, 4046.

(2) Cheung, T. C.; Cheung, K. K.; Peng, S. M.; Che, C. M. J. Chem. Soc., Dalton Trans. 1996, 1645.

(3) For example, see: (a) Roundhill, D. M.; Gray H. B.; Che, C. M. Acc. Chem. Res. 1989, 22, 55. (b) Houlding, V. H.; Miskowski, V. M. Coord. Chem. Rev. 1991, 111, 145. (c) Kunkely, H.; Vogler, A. J. Am. Chem. Soc. 1990, 112, 5625. (d) Cummings, S. D.; Eisenberg, R. J. Am. Chem. Soc. 1996, 118, 1949. (e) Sandrini, D.; Maestri, M.; Balzani, V.; Chassot, L.; von Zelewsky, A. J. Am. Chem. Soc. 1987, 109, 7720 .

(4) For example, see: (a) Peyratout, C. S.; Aldridge, T. K.; Crites, D. K.; McMillin, D. R. Inorg. Chem. 1995, 34, 4484. (b) Kunugi, Y.; Mann, K. R.; Miller, L. L.; Exstrom, C. L. J. Am. Chem. Soc. 1998, 120 589. (c) Wong, K. H.; Chan, M. C. W.; Che, C. M. Chem. Eur. J. 1999, $5,2845$.

(5) Maestri, M.; Sandrini, D.; Balzani, V.; von Zelewsky, A.; Jolliet, P. Helv. Chim. Acta 1988, 71, 134. excited states. ${ }^{8,9}$ The ability of cyclometalated Pd(II) complexes to undergo DNA cleavage has been described. ${ }^{10,11}$

We have previously studied various aspects of luminescent cyclometalated Pt(II) complexes bearing substituted 6-phenyl2,2'-bipyridine ligands. ${ }^{1,2,4 c, 12}$ As part of a continuing program dedicated to luminescent $\mathrm{d}^{8}$ systems, the preparation, crystal structures, and spectroscopic behavior of mono- and binuclear cyclometalated Pd(II) derivatives are presented. The correlation between $\mathrm{Pd}(\mathrm{II})$ and $\mathrm{Pt}(\mathrm{II})$ congeners has enabled evaluation of

(6) (a) Schwarz, R.; Gliemann, G.; Jolliet, P.; von Zelewsky, A. Inorg Chem. 1989, 28, 742. (b) Glasbeek, M.; Sitters, R.; van Veldhoven, E.; von Zelewsky, A.; Humbs, W.; Yersin, H. Inorg. Chem. 1998, 37, 5159.

(7) García-Herbosa, G.; Muñoz, A.; Maestri, M. J. Photochem. Photobiol. A: Chem. 1994, 83, 165.

(8) (a) Maestri, M.; Sandrini, D.; Balzani, V.; von Zelewsky, A.; Cornioley-Deuschel, C.; Jolliet, P. Helv. Chim. Acta 1988, 71, 1053. (b) Barigelletti, F.; Sandrini, D.; Maestri, M.; Balzani, V.; von Zelewsky, A.; Chassot, L.; Jolliet, P.; Maeder, U. Inorg. Chem. 1988. 27, 3644. (c) Maestri, M.; Cornioley-Deuschel, C.; von Zelewsky, A. Coord. Chem. Rev. 1991, 111, 117. (d) Jolliet, P.; Gianini, M.; von Zelewsky, A.; Bernardinelli, G.; Stoeckli-Evans, H. Inorg. Chem. 1996, 35,4883 , and references therein.

(9) Van Houten, K. A.; Heath, D. C.; Barringer, C. A.; Rheingold, A. L.; Pilato, R. S. Inorg. Chem. 1998, 37, 4647.

(10) Newkome, G. R.; Onishi, M.; Puckett, W. E.; Deutsch, W. A. J. Am. Chem. Soc. 1980, 102, 4551.

(11) (a) Suggs, J. W.; Dube, M. J.; Nichols, M. J. Chem. Soc., Chem Commun. 1993, 307. (b) Suggs, J. W.; Higgins, J. D., III; Wagner, R. W.; Millard, J. T. In Metal-DNA Chemistry, ACS Symposium Series 402; Tullius, T. D., Ed.; American Chemical Society: Washington DC, 1989; p 146.

(12) (a) Tse, M. C.; Cheung, K. K.; Chan, M. C. W.; Che, C. M. Chem. Commun. 1998, 2295. (b) Lai, S. W.; Chan, M. C. W.; Cheung, K. K.; Che, C. M. Organometallics 1999, 18, 3327. 
metal-metal and ligand-ligand interactions in these systems and offered insight into their excited-state properties.

\section{Experimental Section}

General Procedures. $\mathrm{K}_{2} \mathrm{PdCl}_{4}$ (Strem), bis(diphenylphosphino)methane, and 1,5-bis(diphenylphosphino)pentane (dppm and $\mathrm{dppC}_{5}$, respectively; Aldrich) were used as received. 6-Phenyl-2,2'-bipyridine and its derivatives were prepared by literature methods. ${ }^{13}$ (Caution! perchlorate salts are potentially explosive and should be handled with care and in small amounts.) Details of solvent treatment for photophysical studies, instrumentation, and emission measurements have been provided previously. ${ }^{1}$

Syntheses. $\left[\mathrm{Pd}\left(\mathrm{L}^{1-5}\right) \mathrm{Cl}\right]\left(1 \mathrm{a}-5 \mathrm{a}, \mathrm{HL}^{1-5}=4\right.$-(aryl)-6-phenyl-2,2'bipyridine; aryl = H (1), phenyl (2), 4-chlorophenyl (3), 4-tolyl (4), 4-methoxyphenyl (5)). A modification of Constable's method was used. ${ }^{14} \mathrm{~A}$ mixture of $\mathrm{K}_{2} \mathrm{PdCl}_{4}(0.16 \mathrm{~g}, 0.49 \mathrm{mmol})$ and $\mathrm{HL}^{1-5}$ (except $\left.\mathrm{L}^{3}, 0.49 \mathrm{mmol}\right)$ in $\mathrm{CH}_{3} \mathrm{CN} / \mathrm{H}_{2} \mathrm{O}(15 / 15 \mathrm{~mL})$ was refluxed for $18 \mathrm{~h}$ to give a yellow suspension, which was then evaporated to dryness. The product was extracted with dichloromethane, and the volume of extract was reduced to $\sim 5 \mathrm{~mL}$. Addition of diethyl ether yielded a yellow solid, which was recrystallized by vapor diffusion of diethyl ether into an acetonitrile solution to afford a yellow crystalline solid.

1a. Yield: $0.16 \mathrm{~g}, 87 \%$. Anal. Calcd for $\mathrm{C}_{16} \mathrm{H}_{11} \mathrm{~N}_{2} \mathrm{PdCl}$ : C, 51.50; H, 2.97; N, 7.51. Found: C, 51.60; H, 2.85; N, 7.50. MS (+ve FAB): $m / z, 372\left(\mathbf{M}^{+}\right), 337\left(\mathrm{M}^{+}-\mathrm{Cl}\right) .{ }^{1} \mathrm{H}$ NMR (DMSO- $\left.d_{6}\right): \delta 7.09(\mathrm{~m}, 2 \mathrm{H})$, $7.52(\mathrm{~d}, 1 \mathrm{H}, J=7.1 \mathrm{~Hz}), 7.62(\mathrm{~d}, 1 \mathrm{H}, J=7.2 \mathrm{~Hz}), 7.78(\mathrm{~m}, 1 \mathrm{H}), 7.99$ $(\mathrm{d}, 1 \mathrm{H}, J=7.9 \mathrm{~Hz}), 8.14(\mathrm{t}, 1 \mathrm{H}, J=7.9 \mathrm{~Hz}), 8.20(\mathrm{~d}, 1 \mathrm{H}, J=7.9$ $\mathrm{Hz}), 8.24(\mathrm{t}, 1 \mathrm{H}, J=7.8 \mathrm{~Hz}), 8.48(\mathrm{~d}, 1 \mathrm{H}, J=8.0 \mathrm{~Hz}), 8.63(\mathrm{~d}, 1 \mathrm{H}$, $J=4.7 \mathrm{~Hz}$ ). ${ }^{13} \mathrm{C}\left\{{ }^{1} \mathrm{H}\right\}$ NMR (DMSO- $\left.d_{6}\right): \delta 119.7,119.8,123.1,124.5$, 124.7, 127.4, 129.6, 136.1, 140.1, 140.4, 148.0, 149.0, 153.5, 154.0, 155.1, 163.6.

2a. Yield: $0.19 \mathrm{~g}, 85 \%$. Anal. Calcd for $\mathrm{C}_{22} \mathrm{H}_{15} \mathrm{~N}_{2} \mathrm{PdCl}$ : C, 58.82; H, 3.37; N, 6.24. Found: C, 59.05; H, 3.25; N, 6.32. MS (+ve FAB): $\mathrm{m} / \mathrm{z} 448\left(\mathrm{M}^{+}\right) .{ }^{1} \mathrm{H}$ NMR $\left(\mathrm{CD}_{3} \mathrm{CN}\right): \delta 7.12(\mathrm{~m}, 2 \mathrm{H}), 7.59-7.66(\mathrm{~m}$, $5 \mathrm{H}), 7.94(\mathrm{~m}, 2 \mathrm{H}), 8.00(\mathrm{~m}, 1 \mathrm{H}), 8.15(\mathrm{~m}, 2 \mathrm{H}), 8.33(\mathrm{~d}, 1 \mathrm{H}, J=7.9$ $\mathrm{Hz}), 8.73(\mathrm{~m}, 2 \mathrm{H}) .{ }^{13} \mathrm{C}\left\{{ }^{1} \mathrm{H}\right\}$ NMR (DMSO- $\left.d_{6}\right): \delta 117.9,118.3,124.4$, $125.6,125.9,128.4,128.5,130.0,130.5,131.3,137.0,141.2,149.0$, 149.9, 152.0, 154.8, 155.0, 156.2, 164.7 .

3a. A mixture of $\mathrm{K}_{2} \mathrm{PdCl}_{4}(0.16 \mathrm{~g}, 0.49 \mathrm{mmol})$ in $\mathrm{H}_{2} \mathrm{O}(10 \mathrm{~mL})$ and $\mathrm{HL}^{3}(0.17 \mathrm{~g}, 0.50 \mathrm{mmol})$ in $\mathrm{CH}_{3} \mathrm{CN}(40 \mathrm{~mL})$ was refluxed for $30 \mathrm{~h}$ to yield an orange suspension, which was filtered and washed with water $(3 \times 20 \mathrm{~mL})$ and diethyl ether $(3 \times 20 \mathrm{~mL})$. Extraction using dimethylformamide (DMF) and recrystallization by diffusion of diethyl ether into a DMF solution gave a yellow solid. Yield: $0.16 \mathrm{~g}, 67 \%$. Anal. Calcd for $\mathrm{C}_{22} \mathrm{H}_{14} \mathrm{~N}_{2} \mathrm{PdCl}_{2}$ : C, 54.63; H, 2.92; N, 5.79. Found: C, 54.90; H, 3.10; N, 5.95. MS (+ve FAB): $m / z, 482\left(\mathrm{M}^{+}\right) .{ }^{1} \mathrm{H}$ NMR $\left(\right.$ DMSO- $\left.d_{6}\right): \delta 7.03-7.12(\mathrm{~m}, 2 \mathrm{H}), 7.52(\mathrm{br} \mathrm{d}, 1 \mathrm{H}, J=6.7 \mathrm{~Hz}), 7.68$ $(\mathrm{d}, 2 \mathrm{H}, J=8.6 \mathrm{~Hz}), 7.76-7.84(\mathrm{~m}, 2 \mathrm{H}), 8.16(\mathrm{~d}, 2 \mathrm{H}, J=8.6 \mathrm{~Hz})$, $8.24-8.31(\mathrm{~m}, 2 \mathrm{H}), 8.54(\mathrm{~s}, 1 \mathrm{H}), 8.63(\mathrm{br} \mathrm{d}, 1 \mathrm{H}, J=4.3 \mathrm{~Hz}), 8.72(\mathrm{~d}$, $1 \mathrm{H}, J=8.0 \mathrm{~Hz}$ ). ${ }^{13} \mathrm{C}\left\{{ }^{1} \mathrm{H}\right\}$ NMR (DMSO- $d_{6}$ ): $\delta 116.9,117.2,123.5$, 124.5, 125.0, 127.4, 129.0, 129.4, 129.6, 134.8, 135.3, 136.1, 140.2, 148.0, 149.0, 149.6, 153.9, 154.1, 155.2, 163.8 .

4a. Yield: $0.17 \mathrm{~g}, 75 \%$. Anal. Calcd for $\mathrm{C}_{23} \mathrm{H}_{17} \mathrm{~N}_{2} \mathrm{PdCl}$ : C, 59.63; H, 3.70; N, 6.05. Found: C, 59.70; H, 3.85; N, 6.12. MS (+ve FAB): $m / z, 462\left(\mathrm{M}^{+}\right) .{ }^{1} \mathrm{H}$ NMR (DMSO- $\left.d_{6}\right): \delta 2.42(\mathrm{~s}, 3 \mathrm{H}, \mathrm{Me}), 7.09(\mathrm{~m}$, $2 \mathrm{H}), 7.42(\mathrm{~d}, 2 \mathrm{H}, J=7.7 \mathrm{~Hz}), 7.53(\mathrm{~d}, 1 \mathrm{H}, J=6.4 \mathrm{~Hz}), 7.79-7.86$ $(\mathrm{m}, 2 \mathrm{H}), 8.04(\mathrm{~d}, 2 \mathrm{H}, J=7.8 \mathrm{~Hz}), 8.30(\mathrm{~m}, 2 \mathrm{H}), 8.53(\mathrm{~s}, 1 \mathrm{H}), 8.65(\mathrm{br}$ $\mathrm{d}, 1 \mathrm{H}, J=4.2 \mathrm{~Hz}), 8.74(\mathrm{~d}, 1 \mathrm{H}, J=7.6 \mathrm{~Hz}) .{ }^{13} \mathrm{C}\left\{{ }^{1} \mathrm{H}\right\}$ NMR (DMSO$\left.d_{6}\right): \delta 20.9(\mathrm{Me}), 116.6,116.9,123.4,124.6,124.8,127.3,127.4,129.5$, $129.7,133.3,136.2,140.2,140.3,148.2,149.1,151.2,153.9,154.1$, 155.5, 164.0.

5a. Yield 0.18 g, 77\%. Anal. Calcd for $\mathrm{C}_{23} \mathrm{H}_{17} \mathrm{~N}_{2} \mathrm{OPdCl}$ : C, 57.64; H, 3.58; N, 5.84. Found: C, 57.70; H, 3.65; N, 5.90. MS (+ve FAB): $m / z, 478\left(\mathrm{M}^{+}\right) .{ }^{1} \mathrm{H}$ NMR $\left(\mathrm{DMSO}-d_{6}\right): \delta 3.88\left(\mathrm{~s}, 3 \mathrm{H}, \mathrm{OCH}_{3}\right), 7.04-$ $7.17(\mathrm{~m}, 4 \mathrm{H}), 7.52(\mathrm{~d}, 1 \mathrm{H}, J=7.0 \mathrm{~Hz}), 7.76-7.86(\mathrm{~m}, 2 \mathrm{H}), 8.13(\mathrm{~d}$, $2 \mathrm{H}, J=8.8 \mathrm{~Hz}), 8.28(\mathrm{~m}, 2 \mathrm{H}), 8.51(\mathrm{~s}, 1 \mathrm{H}), 8.62(\mathrm{~d}, 1 \mathrm{H}, J=5.1 \mathrm{~Hz})$,

(13) Kröhnke, F. Synthesis 1976, 1.

(14) Constable, E. C.; Henney, R. P. G.; Leese, T. A.; Tocher, D. A. J. Chem. Soc. Chem. Commun. 1990, 513. $8.74(\mathrm{~d}, 1 \mathrm{H}, J=8.0 \mathrm{~Hz}) \cdot{ }^{13} \mathrm{C}\left\{{ }^{1} \mathrm{H}\right\} \mathrm{NMR}\left(\mathrm{DMSO}-d_{6}\right): \delta 55.4\left(\mathrm{OCH}_{3}\right)$, $114.4,115.9,116.4,123.4,124.5,124.9,127.3,128.0,129.1,129.4$, 136.0, 140.2, 148.2, 148.9, 150.4, 153.7, 154.1, 155.3, 161.1, 163.6.

[Pd( $\left.\left(\mathbf{L}^{1}\right) \mathbf{B r}\right]$, 1b. A mixture of $\mathbf{1 a}(0.20 \mathrm{~g}, 0.54 \mathrm{mmol})$ in $\mathrm{CH}_{3} \mathrm{CN}$ $(30 \mathrm{~mL})$ and excess $\mathrm{NaBr}(0.50 \mathrm{~g}, 4.86 \mathrm{mmol})$ in $\mathrm{CH}_{3} \mathrm{OH}(10 \mathrm{~mL})$ was stirred for $24 \mathrm{~h}$ at room temperature to afford a yellow solid, which was filtered and washed with water and diethyl ether. Recrystallization by vapor diffusion of diethyl ether into a dimethylformamide solution afforded a yellow crystalline solid. Yield: $0.14 \mathrm{~g}, 63 \%$. Anal. Calcd for $\mathrm{C}_{16} \mathrm{H}_{11} \mathrm{~N}_{2} \mathrm{PdBr}$ : C, 46.02; H, 2.66; N, 6.71. Found: C, 46.10; $\mathrm{H}$, 2.70; N, 6.70. MS (+ve FAB): $m / z 417\left(\mathrm{M}^{+}\right) .{ }^{1} \mathrm{H}$ NMR (DMSO- $\left.d_{6}\right)$ : $\delta 7.01-7.11(\mathrm{~m}, 2 \mathrm{H}), 7.63(\mathrm{~d}, 1 \mathrm{H}, J=7.3 \mathrm{~Hz}), 7.73-7.79(\mathrm{~m}, 2 \mathrm{H})$, $8.00(\mathrm{~d}, 1 \mathrm{H}, J=7.4 \mathrm{~Hz}), 8.13-8.27(\mathrm{~m}, 3 \mathrm{H}), 8.48(\mathrm{~d}, 1 \mathrm{H}, J=8.0$ $\mathrm{Hz}), 8.78(\mathrm{~d}, 1 \mathrm{H}, J=4.7 \mathrm{~Hz}) .{ }^{13} \mathrm{C}\left\{{ }^{1} \mathrm{H}\right\}$ NMR (DMSO- $\left.d_{6}\right): \delta 119.8$, $119.9,123.2$, 124.6, 124.7, 127.5, 129.9, 138.4, 140.2, 140.3, 148.1, $149.9,153.5,153.9,155.3,163.4$.

$\left[\mathbf{P d}\left(\mathbf{L}^{1}\right) \mathbf{I}\right], \mathbf{1 c}$. The procedure for $\mathbf{1 b}$ was adopted using $\mathbf{1 a}(0.20 \mathrm{~g}$, $0.54 \mathrm{mmol})$ and $\mathrm{NaI}(0.50 \mathrm{~g}, 3.34 \mathrm{mmol})$ to give the product as a yellow crystalline solid. Yield: $0.15 \mathrm{~g}, 62 \%$. Anal. Calcd for $\mathrm{C}_{16} \mathrm{H}_{11} \mathrm{~N}_{2} \mathrm{PdI}$ : C, 41.36; H, 2.39; N, 6.03. Found: C, 41.25; H, 2.27; N, 5.90. MS (+ve FAB): $m / z 463\left(\mathrm{M}^{+}\right) .{ }^{1} \mathrm{H}$ NMR (DMSO- $\left.d_{6}\right): \delta 6.97(\mathrm{t}, 1 \mathrm{H}, J=$ $7.1 \mathrm{~Hz}), 7.09(\mathrm{t}, 1 \mathrm{H}, J=6.9 \mathrm{~Hz}), 7.67(\mathrm{~d}, 1 \mathrm{H}, J=6.9 \mathrm{~Hz}), 7.76(\mathrm{t}$, $1 \mathrm{H}, J=6.0 \mathrm{~Hz}), 8.04(\mathrm{~d}, 1 \mathrm{H}, J=6.9 \mathrm{~Hz}), 8.12(\mathrm{~d}, 1 \mathrm{H}, J=7.8 \mathrm{~Hz})$, $8.18-8.26(\mathrm{~m}, 3 \mathrm{H}), 8.50(\mathrm{~d}, 1 \mathrm{H}, J=8.0 \mathrm{~Hz}), 9.03(\mathrm{~d}, 1 \mathrm{H}, J=4.8$ $\mathrm{Hz}) .{ }^{13} \mathrm{C}\left\{{ }^{1} \mathrm{H}\right\}$ NMR (DMSO- $\left.d_{6}\right): \delta 119.9,120.0,123.4,124.3,125.0$, 127.8, 130.4, 134.9, 140.1, 140.3, 148.4, 151.7, 153.4, 153.6, 155.5, 163.1 .

$\left[\mathbf{P d}\left(\mathbf{L}^{1}\right) \mathbf{P P h}_{3}\right] \mathbf{C l O}_{4}, \mathbf{1 d}\left(\mathrm{ClO}_{4}\right)$. A mixture of $\mathbf{1 a}(0.10 \mathrm{~g}, 0.27 \mathrm{mmol})$ and $\mathrm{PPh}_{3}(0.07 \mathrm{~g}, 0.27 \mathrm{mmol})$ in $\mathrm{CH}_{3} \mathrm{CN} / \mathrm{CH}_{3} \mathrm{OH}(20 / 20 \mathrm{~mL})$ was stirred for $3 \mathrm{~h}$ at room temperature. Excess $\mathrm{LiClO}_{4}(0.20 \mathrm{~g}, 1.63 \mathrm{mmol})$ was added to the resultant mixture, which was stirred at room temperature for $5 \mathrm{~h}$ and then filtered and concentrated to $\sim 5 \mathrm{~mL}$. Addition of diethyl ether yielded a yellow solid, which was filtered and washed with diethyl ether. Recrystallization by vapor diffusion of diethyl ether into an acetonitrile solution gave a yellow crystalline solid. Yield: $0.15 \mathrm{~g}, 80 \%$. Anal. Calcd for $\mathrm{C}_{34} \mathrm{H}_{26} \mathrm{~N}_{2} \mathrm{O}_{4} \mathrm{PdClP}$ : C, 58.39; H, 3.75; N, 4.01 . Found: C, 58.20; H, 3.70; N, 3.95. MS (+ve FAB): $\mathrm{m} / z 600\left(\mathrm{M}^{+}\right)$. ${ }^{1} \mathrm{H}$ NMR (DMSO- $\left.d_{6}\right): \delta 6.27(\mathrm{~d}, 1 \mathrm{H}, J=5.2 \mathrm{~Hz}), 6.34(\mathrm{~m}, 1 \mathrm{H}), 6.65$ (t, $1 \mathrm{H}, J=7.3 \mathrm{~Hz}), 7.05-7.18(\mathrm{~m}, 3 \mathrm{H}), 7.57-7.71(\mathrm{~m}, 8 \mathrm{H}), 7.81-$ $7.93(\mathrm{~m}, 6 \mathrm{H}), 8.12-8.35(\mathrm{~m}, 4 \mathrm{H}), 8.54(\mathrm{~d}, 1 \mathrm{H}, J=8.0 \mathrm{~Hz}), 8.74(\mathrm{br}$ s, $1 \mathrm{H}$ ). ${ }^{13} \mathrm{C}\left\{{ }^{1} \mathrm{H}\right\}$ NMR (DMSO- $d_{6}$ ): $\delta 120.4,120.6,123.9,125.9,126.0$, 126.4, 128.3, 128.7, 129.4, 129.9, 132.0, 134.9, 138.2, 138.3, 141.1, $142.4,149.6,149.8,150.2,152.8,157.0,162.2 .{ }^{31} \mathrm{P}\left\{{ }^{1} \mathrm{H}\right\} \mathrm{NMR}$ $\left(\mathrm{CD}_{3} \mathrm{CN}\right): \delta 40.38$.

$\left[\mathbf{P d}_{2}\left(\mathrm{~L}^{1-5}\right)_{2}(\mu\right.$-dppm $\left.)\right]\left(\mathrm{ClO}_{4}\right)_{2}, \mathbf{1 e - 5 e}\left(\mathrm{ClO}_{4}\right)_{2}$. A mixture of $\left[\mathrm{PdL}^{1-5} \mathrm{Cl}\right]$ $(0.32 \mathrm{mmol})$ and dppm $(0.16 \mathrm{mmol})$ in $\mathrm{CH}_{3} \mathrm{CN} / \mathrm{CH}_{3} \mathrm{OH}(15 / 15 \mathrm{~mL})$ was stirred for $12 \mathrm{~h}$ under a nitrogen atmosphere. Excess $\mathrm{LiClO}_{4}$ was added and the resulting solution was concentrated to $\sim 5 \mathrm{~mL}$. Addition of diethyl ether afforded a yellow solid, which was filtered and washed with water $(3 \times 20 \mathrm{~mL})$ and diethyl ether $(2 \times 20 \mathrm{~mL})$. Recrystallization by vapor diffusion of diethyl ether into an acetonitrile solution afforded yellow crystals.

1e( $\left(\mathrm{ClO}_{4}\right)_{2}$. Yield: $0.16 \mathrm{~g}, 79 \%$. Anal. Calcd for $\mathrm{C}_{57} \mathrm{H}_{44} \mathrm{~N}_{4} \mathrm{O}_{8} \mathrm{Pd}_{2-}$ $\mathrm{Cl}_{2} \mathrm{P}_{2}$ : C, 54.39; H, 3.52; N, 4.45. Found: C, 54.52; H, 3.38; N, 4.32. MS (+ve FAB): $m / z 1157\left(\mathrm{M}^{+}+\mathrm{ClO}_{4}\right) .{ }^{1} \mathrm{H}$ NMR $\left(\mathrm{CD}_{3} \mathrm{CN}\right): \delta 4.60$ $\left(\mathrm{t}, 2 \mathrm{H},{ }^{2} J(\mathrm{PH})=12.6 \mathrm{~Hz}, \mathrm{PCH}_{2} \mathrm{P}\right), 6.02(\mathrm{~d}, 2 \mathrm{H}, J=5.2 \mathrm{~Hz}), 6.28(\mathrm{t}$, $2 \mathrm{H}, J=7.4 \mathrm{~Hz}), 6.48(\mathrm{t}, 2 \mathrm{H}, J=7.4 \mathrm{~Hz}), 6.68-6.75(\mathrm{~m}, 4 \mathrm{H}), 6.98$ (d, 2H, $J=7.7 \mathrm{~Hz}), 7.39-7.47(\mathrm{~m}, 10 \mathrm{H}), 7.55-7.59(\mathrm{~m}, 4 \mathrm{H}), 7.75-$ $7.98(\mathrm{~m}, 16 \mathrm{H}) .{ }^{13} \mathrm{C}\left\{{ }^{1} \mathrm{H}\right\}$ NMR $\left(\mathrm{CD}_{3} \mathrm{CN}\right): \delta 21.3\left(\mathrm{t},{ }^{1} \mathrm{~J}(\mathrm{PC})=22 \mathrm{~Hz}\right.$, $\left.\mathrm{PCH}_{2} \mathrm{P}\right), 121.0,124.1,127.2,127.8,130.4,131.1,133.2,134.5,139.9$, $140.9,143.0,150.0,151.5,152.3,153.7,157.1,163.2 .{ }^{31} \mathrm{P}\left\{{ }^{1} \mathrm{H}\right\} \mathrm{NMR}$ $\left(\mathrm{CD}_{3} \mathrm{CN}\right): \delta 35.95$.

2e(ClO $)_{2}$. Yield: $0.18 \mathrm{~g}, 78 \%$. Anal. Calcd for $\mathrm{C}_{69} \mathrm{H}_{52} \mathrm{~N}_{4} \mathrm{O}_{8} \mathrm{Pd}_{2-}$ $\mathrm{Cl}_{2} \mathrm{P}_{2}$ : C, 58.74; H, 3.71; N, 3.97. Found: C, 58.50; H, 3.65; N, 3.92. MS (+ve FAB): $m / z 1311\left(\mathrm{M}^{+}+\mathrm{ClO}_{4}\right) .{ }^{1} \mathrm{H}$ NMR $\left(\mathrm{CD}_{3} \mathrm{CN}\right): \delta 4.63$ $\left(\mathrm{t}, 2 \mathrm{H},{ }^{2} J(\mathrm{PH})=12.7 \mathrm{~Hz}, \mathrm{PCH}_{2} \mathrm{P}\right), 6.07(\mathrm{~d}, 2 \mathrm{H}, J=5.2 \mathrm{~Hz}), 6.31(\mathrm{t}$, $2 \mathrm{H}, J=7.2 \mathrm{~Hz}), 6.49(\mathrm{t}, 2 \mathrm{H}, J=7.5 \mathrm{~Hz}), 6.74-6.81(\mathrm{~m}, 4 \mathrm{H}), 7.19$ $(\mathrm{d}, 2 \mathrm{H}, J=7.1 \mathrm{~Hz}), 7.37-7.61(\mathrm{~m}, 26 \mathrm{H}), 7.86(\mathrm{t}, 4 \mathrm{H}, J=7.9 \mathrm{~Hz})$, $7.94(\mathrm{~s}, 2 \mathrm{H}), 8.12(\mathrm{~d}, 6 \mathrm{H}, J=8.0 \mathrm{~Hz}) .{ }^{13} \mathrm{C}\left\{{ }^{1} \mathrm{H}\right\} \mathrm{NMR}\left(\mathrm{CD}_{3} \mathrm{CN}\right): \delta$ $18.9\left(\mathrm{t},{ }^{1} \mathrm{~J}(\mathrm{PC})=22 \mathrm{~Hz}, \mathrm{PCH}_{2} \mathrm{P}\right), 121.5,124.6,124.7,125.1,125.8$, 
Table 1. Crystal Data

\begin{tabular}{|c|c|c|c|}
\hline & $\mathbf{1 d}\left(\mathrm{ClO}_{4}\right)$ & $\mathbf{1 e}\left(\mathrm{ClO}_{4}\right)_{2} \cdot \mathrm{DMF}$ & $2 \mathrm{e}\left(\mathrm{ClO}_{4}\right)_{2}$ \\
\hline formula & $\mathrm{C}_{34} \mathrm{H}_{26} \mathrm{~N}_{2} \mathrm{O}_{4} \mathrm{PdClP}$ & $\mathrm{C}_{57} \mathrm{H}_{44} \mathrm{~N}_{4} \mathrm{O}_{8} \mathrm{Pd}_{2} \mathrm{Cl}_{2} \mathrm{P}_{2} \cdot\left(\mathrm{CH}_{3}\right)_{2} \mathrm{NCHO}$ & $\mathrm{C}_{69} \mathrm{H}_{52} \mathrm{~N}_{4} \mathrm{O}_{8} \mathrm{Pd}_{2} \mathrm{Cl}_{2} \mathrm{P}_{2}$ \\
\hline fw & 699.44 & 1331.78 & 1410.83 \\
\hline crystal system & monoclinic & monoclinic & monoclinic \\
\hline space group & $P 2_{1} / c$ & $P 2_{1} / c$ & $P 2_{1} / c$ \\
\hline color & yellow & yellow & yellow \\
\hline crystal size, $\mathrm{mm}$ & $0.15 \times 0.25 \times 0.30$ & $0.05 \times 0.25 \times 0.25$ & $0.30 \times 0.30 \times 0.45$ \\
\hline$a, \AA$ & $9.801(1)$ & $15.602(2)$ & $14.331(3)$ \\
\hline$b, \AA$ & $20.122(1)$ & $13.253(3)$ & $14.535(3)$ \\
\hline$c, \AA$ & $15.205(1)$ & $27.213(3)$ & $31.901(8)$ \\
\hline$\beta, \operatorname{deg}$ & $98.096(9)$ & $98.70(2)$ & $93.55(4)$ \\
\hline$V, \AA^{3}$ & $2968.5(5)$ & $5562(1)$ & $6632(3)$ \\
\hline$Z$ & 4 & 4 & 4 \\
\hline$D_{\mathrm{c}}, \mathrm{g} \mathrm{cm}^{-3}$ & 1.565 & 1.590 & 1.413 \\
\hline$\mu, \mathrm{cm}^{-1}$ & 8.12 & 8.64 & 7.09 \\
\hline$F(000)$ & 1416 & 2696 & 2849 \\
\hline $2 \theta_{\max }, \mathrm{deg}$ & 50 & 48 & 45 \\
\hline$R,{ }^{a} R_{\mathrm{W}}^{b}$ & $0.040,0.056$ & $0.071,0.087$ & $0.065,0.084$ \\
\hline residual $\rho$, e $\AA^{-3}$ & $-0.42,+1.02$ & $-0.73,+1.18$ & $-1.12,+1.05$ \\
\hline
\end{tabular}

127.6, 128.5, 129.1, 129.5, 129.8, 130.5, 133.9, 137.2, 138.1, 147.3, 148.9, 149.6, 151.3, 151.8, 154.4, 160.7. ${ }^{31} \mathrm{P}\left\{{ }^{1} \mathrm{H}\right\}$ NMR $\left(\mathrm{CD}_{3} \mathrm{CN}\right): \delta$ 36.66.

3e( $\left(\mathrm{ClO}_{4}\right)_{2}$. Yield: $0.15 \mathrm{~g}, 65 \%$. Anal. Calcd for $\mathrm{C}_{69} \mathrm{H}_{50} \mathrm{~N}_{4} \mathrm{O}_{8} \mathrm{Pd}_{2}-$ $\mathrm{Cl}_{4} \mathrm{P}_{2}$ : C, 56.01; $\mathrm{H}, 3.41 ; \mathrm{N}, 3.79$. Found: C, 55.88; H, 3.28; $\mathrm{N}, 3.65$. MS (+ve FAB): $m / z 1380\left(\mathrm{M}^{+}+\mathrm{ClO}_{4}\right) .{ }^{1} \mathrm{H}$ NMR $\left(\mathrm{CD}_{3} \mathrm{CN}\right): \delta 4.61$ $\left(\mathrm{t}, 2 \mathrm{H},{ }^{2} J(\mathrm{PH})=12.7 \mathrm{~Hz}, \mathrm{PCH}_{2} \mathrm{P}\right), 6.02(\mathrm{~m}, 2 \mathrm{H}), 6.28(\mathrm{~m}, 2 \mathrm{H}), 6.40$ (t, 2H, $J=7.4 \mathrm{~Hz}), 6.79(\mathrm{~m}, 4 \mathrm{H}), 7.21(\mathrm{~d}, 2 \mathrm{H}, J=7.6 \mathrm{~Hz}), 7.33-$ $8.22(\mathrm{~m}, 36 \mathrm{H}) .{ }^{13} \mathrm{C}\left\{{ }^{1} \mathrm{H}\right\}$ NMR $\left(\mathrm{CD}_{3} \mathrm{CN}\right): \delta 21.2\left(\mathrm{t},{ }^{1} J(\mathrm{PC})=22 \mathrm{~Hz}\right.$, $\left.\mathrm{PCH}_{2} \mathrm{P}\right), 116.7,123.3,126.3,126.5,126.9,129.0,129.3,129.4,130.4$, 130.9, 131.2, 131.6, 132.3, 134.0, 136.8, 138.9, 139.8, 148.9, 150.4, 151.3, 152.0, 153.1, 156.2, 162.6. ${ }^{31} \mathrm{P}\left\{{ }^{1} \mathrm{H}\right\}$ NMR $\left(\mathrm{CD}_{3} \mathrm{CN}\right): \delta 36.64$.

4e( $\left(\mathrm{ClO}_{4}\right)_{2}$. Yield: $0.16 \mathrm{~g}, 70 \%$. Anal. Calcd for $\mathrm{C}_{71} \mathrm{H}_{56} \mathrm{~N}_{4} \mathrm{O}_{8} \mathrm{Pd}_{2}-$ $\mathrm{Cl}_{2} \mathrm{P}_{2}$ : C, 59.26; H, 3.92; N, 3.89. Found: C, 59.20; H, 3.85; N, 3.73. MS (+ve FAB): $1339\left(\mathrm{M}^{+}+\mathrm{ClO}_{4}\right) .{ }^{1} \mathrm{H}$ NMR $\left(\mathrm{CD}_{3} \mathrm{CN}\right): \delta 2.42(\mathrm{~s}$, $6 \mathrm{H}, \mathrm{Me}), 4.61\left(\mathrm{t}, 2 \mathrm{H},{ }^{2} J(\mathrm{PH})=12.7 \mathrm{~Hz}, \mathrm{PCH}_{2} \mathrm{P}\right), 6.04(\mathrm{~d}, 2 \mathrm{H}, J=5.0$ $\mathrm{Hz}), 6.30(\mathrm{t}, 2 \mathrm{H}, J=6.9 \mathrm{~Hz}), 6.45(\mathrm{t}, 2 \mathrm{H}, J=7.3 \mathrm{~Hz}), 6.73-6.80(\mathrm{~m}$, 4H), $7.18(\mathrm{~d}, 6 \mathrm{H}, J=7.8 \mathrm{~Hz}), 7.47-7.55(\mathrm{~m}, 20 \mathrm{H}), 7.82-7.91(\mathrm{~m}$, $6 \mathrm{H}), 8.10(\mathrm{~d}, 6 \mathrm{H}, J=8.0 \mathrm{~Hz}) .{ }^{13} \mathrm{C}\left\{{ }^{1} \mathrm{H}\right\} \mathrm{NMR}\left(\mathrm{CD}_{3} \mathrm{CN}\right): \delta 20.6(\mathrm{Me})$, $20.9\left(\mathrm{t},{ }^{1} J(\mathrm{PC})=22 \mathrm{~Hz}, \mathrm{PCH}_{2} \mathrm{P}\right), 116.4,123.2,126.2,126.4,126.8$, 127.3, 129.4, 129.8, 130.1, 131.0, 131.3, 131.6, 132.2, 132.4, 138.8, $139.7,141.4,149.0,150.5,151.2,152.9,153.1,156.2,162.3 .{ }^{31} \mathrm{P}\left\{{ }^{1} \mathrm{H}\right\}$ NMR $\left(\mathrm{CD}_{3} \mathrm{CN}\right): \delta 36.53$.

5e(ClO $\left.{ }_{4}\right)_{2}$. Yield: 0.17 g, $73 \%$. Anal. Calcd for $\mathrm{C}_{71} \mathrm{H}_{56} \mathrm{~N}_{4} \mathrm{O}_{10} \mathrm{Pd}_{2-}$ $\mathrm{Cl}_{2} \mathrm{P}_{2}$ : C, 57.98; H, 3.84; N, 3.81. Found: C, 57.85; H, 3.75; N, 3.90. MS (+ve FAB): $m / z 1369\left(\mathrm{M}^{+}+\mathrm{ClO}_{4}\right) .{ }^{1} \mathrm{H}$ NMR $\left(\mathrm{CD}_{3} \mathrm{CN}\right): \delta 3.88$ (s, 6H, OMe), $4.59\left(\mathrm{t}, 2 \mathrm{H},{ }^{2} \mathrm{~J}(\mathrm{PH})=12.7 \mathrm{~Hz}, \mathrm{PCH}_{2} \mathrm{P}\right), 6.04(\mathrm{~d}, 2 \mathrm{H}, J$ $=5.2 \mathrm{~Hz}), 6.28(\mathrm{t}, 2 \mathrm{H}, J=7.6 \mathrm{~Hz}), 6.43(\mathrm{t}, 2 \mathrm{H}, J=7.4 \mathrm{~Hz}), 6.73-$ $6.80(\mathrm{~m}, 4 \mathrm{H}), 6.87(\mathrm{~d}, 4 \mathrm{H}, J=8.8 \mathrm{~Hz}), 7.18(\mathrm{~d}, 2 \mathrm{H}, J=7.7 \mathrm{~Hz})$, 7.46-7.60 (m, 20H), 7.78-7.89 (m, 6H), $8.11(\mathrm{~m}, 6 \mathrm{H}) .{ }^{13} \mathrm{C}\left\{{ }^{1} \mathrm{H}\right\} \mathrm{NMR}$ $\left(\mathrm{CD}_{3} \mathrm{CN}\right): \delta 22.1\left(\mathrm{t},{ }^{1} J(\mathrm{PC})=22 \mathrm{~Hz}, \mathrm{PCH}_{2} \mathrm{P}\right), 56.3(\mathrm{OMe}), 115.6$, 117.0, 117.7, 124.1, 127.1, 127.3, 127.8, 128.5, 130.0, 130.4, 131.1, $139.8,140.7,150.2,151.5,152.3,153.9,157.4,163.0,163.3 .{ }^{31} \mathrm{P}\left\{{ }^{1} \mathrm{H}\right\}$ NMR $\left(\mathrm{CD}_{3} \mathrm{CN}\right): \delta 36.29$.

$\left[\mathbf{P d}_{2}\left(\mathrm{~L}^{1}\right)_{2}\left(\mu-\mathrm{dppC}_{5}\right)\right]\left(\mathrm{ClO}_{4}\right)_{2}, \mathbf{1 f}\left(\mathrm{ClO}_{4}\right)_{2}$. The procedure for $1 \mathrm{e}$ was adopted using $1 \mathbf{a}(0.15 \mathrm{~g}, 0.40 \mathrm{mmol})$ and 1,5-bis(diphenylphosphino)pentane $(0.09 \mathrm{~g}, 0.20 \mathrm{mmol})$ to yield a yellow crystalline solid. Yield: $0.19 \mathrm{~g}, 72 \%$. Anal. Calcd for $\mathrm{C}_{61} \mathrm{H}_{52} \mathrm{~N}_{4} \mathrm{O}_{8} \mathrm{Pd}_{2} \mathrm{Cl}_{2} \mathrm{P}_{2}$ : C, 55.72; $\mathrm{H}, 3.99$; N, 4.26. Found: C, 55.68; H, 3.92; N, 4.20. MS (+ve FAB): $\mathrm{m} / \mathrm{z}$ $1215\left(\mathrm{M}^{+}+\mathrm{ClO}_{4}\right) \cdot{ }^{1} \mathrm{H}$ NMR (DMSO- $\left.d_{6}\right): \delta 1.74 ; 1.83 ; 2.70(\mathrm{br}$ $\left.\mathrm{m}, 10 \mathrm{H}, \mathrm{P}\left(\mathrm{CH}_{2}\right)_{5} \mathrm{P}\right), 6.45(\mathrm{~m}, 2 \mathrm{H}), 6.67(\mathrm{~m}, 4 \mathrm{H}), 6.97(\mathrm{t}, 2 \mathrm{H}, J=$ $7.5 \mathrm{~Hz}), 7.14(\mathrm{t}, 2 \mathrm{H}, J=7.0 \mathrm{~Hz}), 7.46(\mathrm{~m}, 8 \mathrm{H}), 7.55(\mathrm{~m}, 4 \mathrm{H}), 7.67$ $(\mathrm{d}, 2 \mathrm{H}, J=7.2 \mathrm{~Hz}), 7.76(\mathrm{~m}, 8 \mathrm{H}), 8.06(\mathrm{~m}, 4 \mathrm{H}), 8.19-8.24(\mathrm{~m}$, $4 \mathrm{H}), 8.41(\mathrm{~d}, 2 \mathrm{H}, J=8.0 \mathrm{~Hz}) .{ }^{13} \mathrm{C}\left\{{ }^{1} \mathrm{H}\right\}$ NMR (DMSO- $\left.d_{6}\right): \delta 22.6$ $\left(\mathrm{d}, J(\mathrm{PC})=30 \mathrm{~Hz}, \mathrm{PCH}_{2}\right), 24.3\left(\mathrm{~d}, J(\mathrm{PC})=4 \mathrm{~Hz}, \mathrm{PCH}_{2} \mathrm{CH}_{2}\right), 30.3$ $\left(\mathrm{t}, J(\mathrm{PC})=16 \mathrm{~Hz}, \mathrm{P}\left(\mathrm{CH}_{2}\right)_{2} \mathrm{CH}_{2}\right), 119.2,119.3,122.7,124.8,125.6$, $128.1,128.7,129.1,129.2,130.5,132.0,136.0,136.1,139.9,141.1$, 148.2, 149.5, 149.6, 151.6, 155.6, 160.9. ${ }^{31} \mathrm{P}\left\{{ }^{1} \mathrm{H}\right\} \mathrm{NMR}\left(\mathrm{CD}_{3} \mathrm{CN}\right): \delta$ 31.51 .
X-ray Crystallography. Crystals of $\mathbf{1 d}\left(\mathrm{ClO}_{4}\right), \mathbf{1 e}\left(\mathrm{ClO}_{4}\right)_{2} \cdot \mathrm{DMF}$, and $\mathbf{2 e}\left(\mathrm{ClO}_{4}\right)_{2}$ were obtained by vapor diffusion of diethyl ether into acetonitrile/dimethylformamide (DMF) solutions. Crystal data and details of collection and refinement are summarized in Table 1. The following data are listed in the order $\mathbf{1 d}\left(\mathrm{ClO}_{4}\right) / \mathbf{1} \mathbf{e}\left(\mathrm{ClO}_{4}\right)_{2} \cdot \mathrm{DMF}$. A total of 5408/9147 unique reflections was collected at $301 \mathrm{~K}$ on a Rigaku/ Nonius diffractometer $[\lambda(\mathrm{Mo}-\mathrm{K} \alpha)=0.7107 \AA, \omega-2 \theta$ scans $]$. The structures were solved by Patterson methods, expanded using Fourier techniques (PATTY ${ }^{15}$ ), and refined by full-matrix least squares using the TeXsan ${ }^{16}$ software package for $4180 / 5692$ absorption-corrected (transmission $0.88-1.00 / 0.83-1.00$ ) reflections with $I>3 \sigma(I)$ and 388/ 689 parameters. For $\mathbf{2 e}\left(\mathrm{ClO}_{4}\right)_{2}$, a total of 8647 unique reflections was collected at $298 \mathrm{~K}$ on a Nonius diffractometer $[\lambda(\mathrm{Mo}-\mathrm{K} \alpha)=0.7107$ $\AA, \theta / 2 \theta$ scans]. The structure was solved by Patterson methods, expanded using Fourier techniques, and refined by least-squares treatment on $F^{2}$ using the NRCVAX program for 4698 absorptioncorrected (transmission $0.65-1.00$ ) reflections with $I>2 \sigma(I)$ and 793 parameters. The pairs of atoms $\mathrm{N}(1) / \mathrm{C}(1)$ for $\mathbf{1 d}\left(\mathrm{ClO}_{4}\right), \mathrm{N}(1) / \mathrm{C}(1)$ and $\mathrm{N}(3) / \mathrm{C}(17)$ for $1 \mathrm{e}\left(\mathrm{ClO}_{4}\right)_{2} \cdot \mathrm{DMF}$, and $\mathrm{N}(1) / \mathrm{C}(22)$ and $\mathrm{N}(3) / \mathrm{C}(44)$ for $2 \mathbf{e}\left(\mathrm{ClO}_{4}\right)_{2}$ were differentiated by their temperature factors; interchanging the respective $\mathrm{C}$ and $\mathrm{N}$ atoms resulted in unreasonable temperature factors and/or higher $R$ values.

\section{Results and Discussion}

Synthesis and Characterization. The complexes described in this study are illustrated in Figure 1. Cyclopalladation of the ligands $\mathrm{HL}^{1-5}$, prepared by Kröhnke syntheses, ${ }^{13}$ is accomplished by modifying the procedure described by Constable and co-workers. ${ }^{14}$ The various 4 -aryl substituents in $\mathrm{HL}^{1-5}$ may be expected to impose different electronic demands upon the palladium center. Hence complexes $\mathbf{1 a}-\mathbf{5 a}$ are synthesized by interaction of $\mathrm{K}_{2} \mathrm{PdCl}_{4}$ with $\mathrm{HL}^{1-5}$ respectively at elevated temperatures in an acetonitrile/water mixture. The coordinated chloride auxiliary in this series allows derivatization of the $\left[\mathrm{Pd}\left(\mathrm{L}^{1-5}\right)\right]$ moieties through ligand-substitution reactions. The neutral complexes $\left[\mathrm{Pd}\left(\mathrm{L}^{1}\right) \mathrm{X}\right](\mathrm{X}=\mathrm{Br}, \mathbf{1 b} ; \mathrm{I}, \mathbf{1 c})$ and the cationic derivative $\left[\mathrm{Pd}\left(\mathrm{L}^{1}\right) \mathrm{PPh}_{3}\right]^{+}(\mathbf{1 d})$ are formed by reaction of $\mathbf{1 a}$ with $\mathrm{NaX}$ and triphenylphosphine respectively at room temperature.

The binuclear cyclometalated $\mathrm{Pd}(\mathrm{II})$ complexes $\mathbf{1 e}-\mathbf{5 e}$ and 1f are conveniently prepared by treatment of the respective

(15) PATTY: Beurskens, P. T.; Admiraal, G.; Beursken, G.; Bosman, W P.; Garcia-Granda, S.; Gould, R. O.; Smits, J. M. M.; Smykalla, C. The DIRDIF program system, Technical Report of the Crystallography Laboratory, University of Nijmegen, The Netherlands, 1992.

(16) TeXsan: Crystal Structure Analysis Package; Molecular Structure Corporation: The Woodlands, TX, 1985 and 1992. 


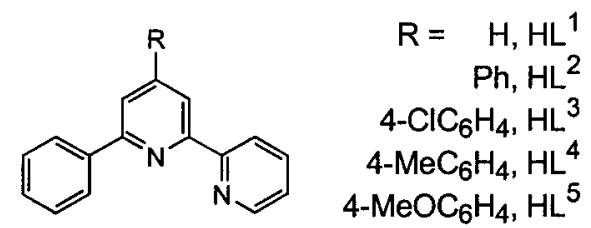

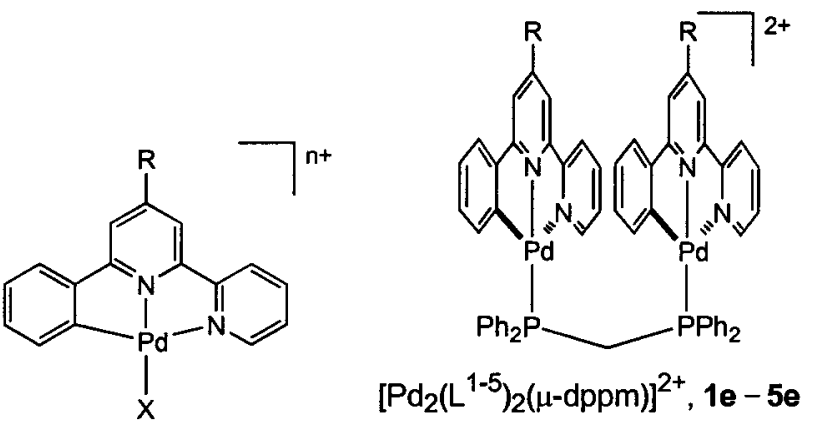

$\operatorname{Pd}\left(L^{1}\right) X ; n=0 ; X=C l, 1 a$

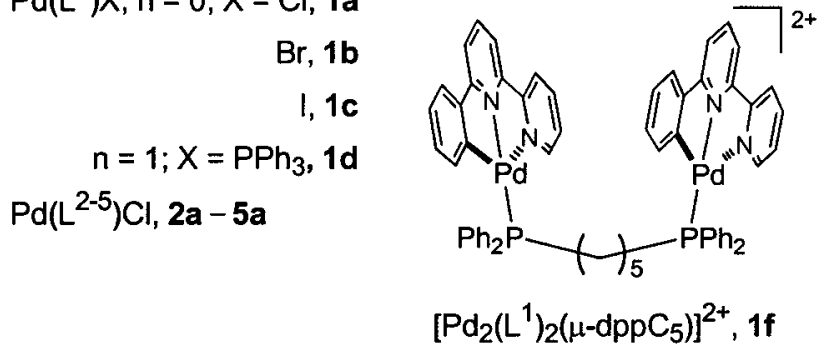

Figure 1. Nomenclature for ligands and complexes.

mononuclear precursor with the appropriate diphosphine ligand. In the ${ }^{1} \mathrm{H}$ NMR spectra, the methylene group of the dppm bridge appears as a distinctive triplet resonance at ca. $4.6 \mathrm{ppm}\left({ }^{2} \mathrm{~J}(\mathrm{PH})\right.$ $=12.7 \mathrm{~Hz}$ ). The positive FAB mass spectra for $\mathbf{1} \mathbf{e}-\mathbf{5 e}$ contain a prominent signal corresponding to the $\left(\mathrm{M}^{+}+\mathrm{ClO}_{4}\right)$ fragment rather than the molecular cation $\mathrm{M}^{+}$.

Crystal Structures: Pd-Pd and $\boldsymbol{\pi}-\boldsymbol{\pi}$ Separations. A number of palladium(II) complexes have been crystallographically characterized during this investigation. Comparisons with the structures of platinum(II) congeners can provide insight into the extent of metal-metal and ligand-ligand interactions in these systems. Selected bond distances and angles for $\mathbf{1} \mathbf{d}\left(\mathrm{ClO}_{4}\right)$, $\mathbf{1 e}\left(\mathrm{ClO}_{4}\right)_{2} \cdot \mathrm{DMF}$, and $\mathbf{2 e}\left(\mathrm{ClO}_{4}\right)_{2}$ are listed in Table 2. Constable and co-workers ${ }^{14}$ have communicated the crystal structure of 1a.

The Pd atom in the cation 1d (Figure 2) resides in a highly distorted square planar environment. The bite angle of $157.6(2)^{\circ}$ exhibited by $\mathrm{L}^{1}$ is typical for cyclometalating 6-phenyl-2,2'-bipyridine ligands. The palladium-ligand distances are comparable to those in the platinum analogue ${ }^{2}$ (e.g., $\mathrm{Pd}-\mathrm{P}$,

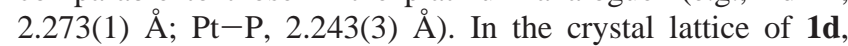
distinct $\pi-\pi$ stacking interactions are not apparent due to poor overlap of $\mathrm{L}^{1}$ ligands, although some close intermolecular contacts $(3.5-3.7 \AA)$ are observed. This is in contrast to the structure of $\left[\mathrm{Pt}\left(\mathrm{L}^{1}\right) \mathrm{PPh}_{3}\right] \mathrm{ClO}_{4},{ }^{2}$ which displays head-to-tail $\pi$-stacking interactions with intermolecular plane separations of $3.35 \AA$. In addition, Jeffrey, Ward, and co-workers ${ }^{17}$ have reported the crystal structure of the $\mathrm{Pd}(\mathrm{II})$ complex $\left[\mathrm{Pd}\left(\mathrm{L}^{\prime}\right) \mathrm{Cl}\right]$ $\left(\mathrm{HL}^{\prime}=6\right.$-(2-hydroxyphenyl)-2,2'-bipyridine), which reveals an average stacking distance of $3.44 \AA$ between the $\mathrm{L}^{\prime}$ planes.

Like the platinum equivalent $\left[\mathrm{Pt}_{2}\left(\mathrm{~L}^{1}\right)_{2}(\mu \text {-dppm })\right]^{2+}, 2$ the molecular structures of the cations $\left[\mathrm{Pd}_{2}\left(\mathrm{~L}^{1}\right)_{2}(\mu \text {-dppm) }]^{2+}(\mathbf{1 e})\right.$

(17) Bardwell, D. A.; Crossley, J. G.; Jeffery, J. C.; Orpen, A. G.; Psillakis, E.; Tilley, E. E. M.; Ward, M. D. Polyhedron, 1994, 13, 2291.

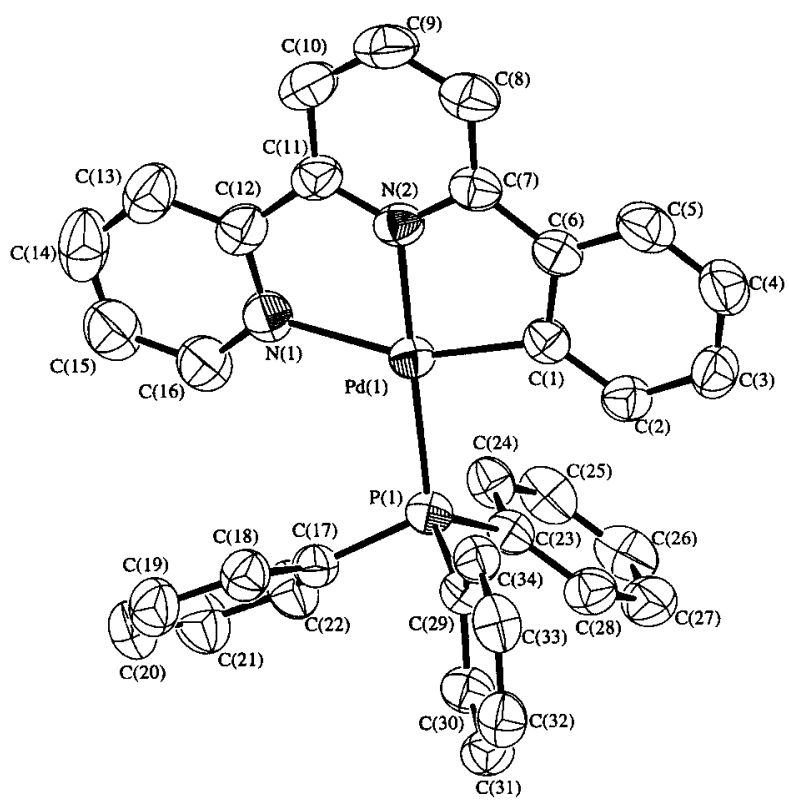

Figure 2. Perspective view of $\left[\mathrm{Pd}\left(\mathrm{L}^{1}\right) \mathrm{PPh}_{3}\right]^{+}, \mathbf{1 d}(50 \%$ probability ellipsoids).

Table 2. Selected Bond Lengths ( $\mathrm{A})$ and Angles (deg)

\begin{tabular}{lclc}
\hline \multicolumn{5}{c}{$\left[\mathrm{Pd}\left(\mathrm{L}^{1}\right) \mathrm{PPh}_{3}\right] \mathrm{ClO}_{4}, \mathbf{1 d}\left(\mathrm{ClO}_{4}\right)$} \\
$\mathrm{Pd}(1)-\mathrm{P}(1)$ & $2.273(1)$ & $\mathrm{Pd}(1)-\mathrm{N}(2)$ & $2.020(4)$ \\
$\mathrm{Pd}(1)-\mathrm{N}(1)$ & $2.191(4)$ & $\mathrm{Pd}(1)-\mathrm{C}(1)$ & $2.039(5)$ \\
$\mathrm{P}(1)-\mathrm{Pd}(1)-\mathrm{N}(1)$ & $106.7(1)$ & $\mathrm{P}(1)-\mathrm{Pd}(1)-\mathrm{C}(1)$ & $95.6(1)$ \\
$\mathrm{P}(1)-\mathrm{Pd}(1)-\mathrm{N}(2)$ & $172.8(1)$ & $\mathrm{N}(1)-\mathrm{Pd}(1)-\mathrm{C}(1)$ & $157.6(2)$ \\
\multicolumn{4}{c}{$\left[\mathrm{Pd}_{2}\left(\mathrm{~L}^{1}\right)_{2}(\mu-\mathrm{dppm})\right]\left(\mathrm{ClO}_{4}\right)_{2} \cdot \mathrm{DMF}, \mathbf{1 e}\left(\mathrm{ClO}_{4}\right)_{2} \cdot \mathrm{DMF}$} \\
$\mathrm{Pd}(1)-\mathrm{P}(1)$ & $2.266(2)$ & $\mathrm{Pd}(2)-\mathrm{P}(2)$ & $2.272(2)$ \\
$\mathrm{Pd}(1)-\mathrm{N}(1)$ & $2.116(9)$ & $\mathrm{Pd}(2)-\mathrm{N}(3)$ & $2.17(1)$ \\
$\mathrm{Pd}(1)-\mathrm{N}(2)$ & $2.025(8)$ & $\mathrm{Pd}(2)-\mathrm{N}(4)$ & $2.055(8)$ \\
$\mathrm{Pd}(1)-\mathrm{C}(1)$ & $2.17(1)$ & $\mathrm{Pd}(2)-\mathrm{C}(17)$ & $2.07(1)$ \\
$\mathrm{P}(1)-\mathrm{C}(45)$ & $1.845(9)$ & $\mathrm{P}(2)-\mathrm{C}(45)$ & $1.829(9)$ \\
$\mathrm{P}(1)-\mathrm{Pd}(1)-\mathrm{N}(1)$ & $102.6(3)$ & $\mathrm{P}(2)-\mathrm{Pd}(2)-\mathrm{N}(3)$ & $105.6(3)$ \\
$\mathrm{P}(1)-\mathrm{Pd}(1)-\mathrm{N}(2)$ & $174.9(3)$ & $\mathrm{P}(2)-\mathrm{Pd}(2)-\mathrm{N}(4)$ & $174.9(2)$ \\
$\mathrm{P}(1)-\mathrm{Pd}(1)-\mathrm{C}(1)$ & $97.4(3)$ & $\mathrm{P}(2)-\mathrm{Pd}(2)-\mathrm{C}(17)$ & $97.4(3)$ \\
$\mathrm{N}(1)-\mathrm{Pd}(1)-\mathrm{C}(1)$ & $160.0(4)$ & $\mathrm{N}(3)-\mathrm{Pd}(2)-\mathrm{C}(17)$ & $156.9(4)$ \\
$\mathrm{Pd}(1)-\mathrm{P}(1)-\mathrm{C}(45)$ & $115.5(3)$ & $\mathrm{Pd}(2)-\mathrm{P}(2)-\mathrm{C}(45)$ & $115.9(3)$ \\
$\mathrm{P}(1)-\mathrm{C}(45)-\mathrm{P}(2)$ & $122.8(5)$ & & \\
\multicolumn{5}{c}{$\left[\begin{array}{ll}\left.\mathrm{Pd}_{2}\left(\mathrm{~L}^{2}\right)_{2}(\mu-\mathrm{dppm})\right](\mathrm{ClO})_{4}, 2 \mathrm{e}(\mathrm{ClO} \\
4\end{array}\right)_{2}$} \\
$\mathrm{Pd}(1)-\mathrm{P}(1)$ & $2.279(5)$ & $\mathrm{Pd}(2)-\mathrm{P}(2)$ & $2.280(5)$ \\
$\mathrm{Pd}(1)-\mathrm{N}(1)$ & $2.182(13)$ & $\mathrm{Pd}(2)-\mathrm{N}(3)$ & $2.177(13)$ \\
$\mathrm{Pd}(1)-\mathrm{N}(2)$ & $2.003(12)$ & $\mathrm{Pd}(2)-\mathrm{N}(4)$ & $1.991(13)$ \\
$\mathrm{Pd}(1)-\mathrm{C}(22)$ & $2.021(16)$ & $\mathrm{Pd}(2)-\mathrm{C}(44)$ & $1.999(17)$ \\
$\mathrm{P}(1)-\mathrm{C}(45)$ & $1.853(17)$ & $\mathrm{P}(2)-\mathrm{C}(45)$ & $1.856(17)$ \\
$\mathrm{P}(1)-\mathrm{Pd}(1)-\mathrm{N}(1)$ & $105.2(4)$ & $\mathrm{P}(2)-\mathrm{Pd}(2)-\mathrm{N}(3)$ & $106.7(4)$ \\
$\mathrm{P}(1)-\mathrm{Pd}(1)-\mathrm{N}(2)$ & $175.6(4)$ & $\mathrm{P}(2)-\mathrm{Pd}(2)-\mathrm{N}(4)$ & $175.7(4)$ \\
$\mathrm{P}(1)-\mathrm{Pd}(1)-\mathrm{C}(22)$ & $96.8(5)$ & $\mathrm{P}(2)-\mathrm{Pd}(2)-\mathrm{C}(44)$ & $95.2(5)$ \\
$\mathrm{N}(1)-\mathrm{Pd}(1)-\mathrm{C}(22)$ & $157.6(6)$ & $\mathrm{N}(3)-\mathrm{Pd}(2)-\mathrm{C}(44)$ & $157.0(6)$ \\
$\mathrm{Pd}(1)-\mathrm{P}(1)-\mathrm{C}(45)$ & $116.1(5)$ & $\mathrm{Pd}(2)-\mathrm{P}(2)-\mathrm{C}(45)$ & $117.1(5)$ \\
$\mathrm{P}(1)-\mathrm{C}(45)-\mathrm{P}(2)$ & $120.5(9)$ & & \\
& & &
\end{tabular}

and $\left[\operatorname{Pd}_{2}\left(\mathrm{~L}^{2}\right)_{2}(\mu \text {-dppm) }]^{2+}(\mathbf{2 e})\right.$ (Figures 3 and 4 respectively) depict two $\left[\operatorname{Pd}\left(\mathrm{L}^{n}\right)\right]$ moieties tethered by a dppm linker. The intramolecular Pd-Pd separations of 3.230(1) $\AA$ in 1e and $3.320(2) \AA$ in $2 \mathbf{e}$ are significantly greater than that expected for a strong Pd-Pd bonding interaction (typically $2.6-2.8 \AA$ )..$^{18}$ Outside this range, longer $\mathrm{Pd}-\mathrm{Pd}$ distances have been observed in $\left[\mathrm{Pd}_{2}\left(\mu_{\mathrm{NS}}, \eta^{2}-\mathrm{L}\right)_{2} \mathrm{Cl}_{2}\left(\mathrm{PMe}_{3}\right)_{2}\right](\mathrm{L}=$ heterocyclic 2-thiolate, 2.915(1)-3.104(2) $)^{1},{ }^{19}\left[\mathrm{Pd}_{2}\left(\mathrm{C}_{6} \mathrm{H}_{4} \mathrm{~N}(\mathrm{H}) \mathrm{N}=\mathrm{C}\left(\mathrm{CH}_{3}\right) \mathrm{C}_{5} \mathrm{H}_{4} \mathrm{~N}\right)_{2}(\mu-\right.$

(18) For example, see: (a) Cotton, F. A.; Matusz, M.; Poli, R.; Feng, X. J. Am. Chem. Soc. 1988, 110, 1144. (b) Ogoshi, S.; Tsutsumi, K.; Ooi, M.; Kurosawa, H. J. Am. Chem. Soc. 1995, 117, 10415. (c) Tanase, T.; Ukaji, H.; Yamamoto, Y. J. Chem. Soc., Dalton Trans. 1996, 3059 . 


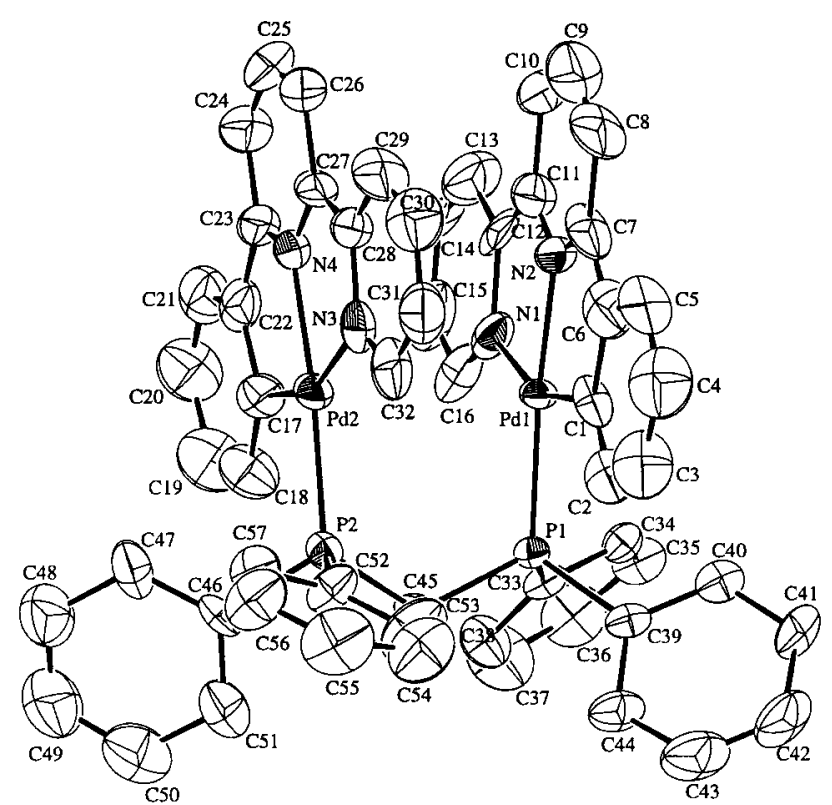

Figure 3. Perspective view of $\left[\mathrm{Pd}_{2}\left(\mathrm{~L}^{1}\right)_{2}(u \text {-dppm) }]^{2+}\right.$, 1e (35\% probability ellipsoids).

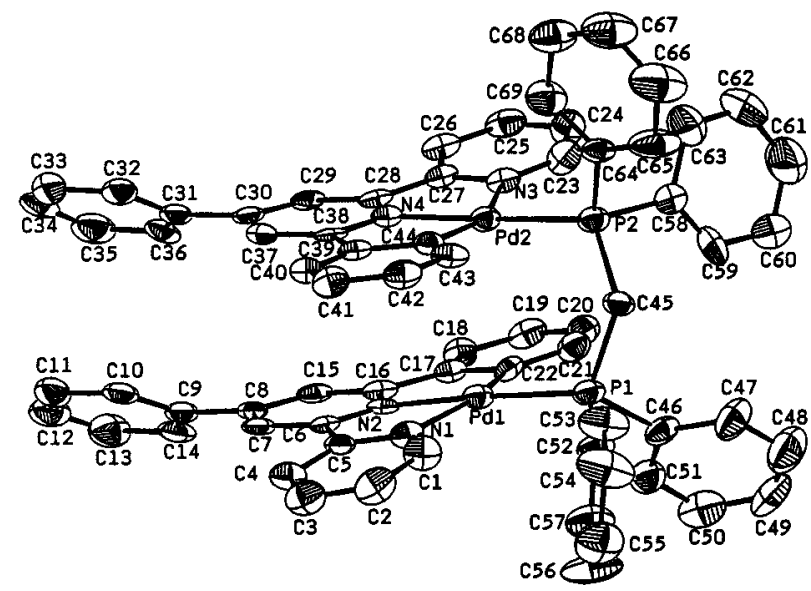

Figure 4. Perspective view of $\left[\mathrm{Pd}_{2}\left(\mathrm{~L}^{2}\right)_{2}(\mu-\mathrm{dppm})\right]^{2+}, \mathbf{2 e}(30 \%$ probability ellipsoids).

$\mathrm{dppm})]\left(\mathrm{BF}_{4}\right)_{2}(3.114(1) \AA),{ }^{20}\left[\mathrm{Pd}_{2}\left(2-\left(5^{\prime}-\mathrm{MeC}_{6} \mathrm{H}_{3}\right)-1,10 \text {-phen }\right)_{2}-\right.$ $(\mu$-N-3,N-9-adenine $)]\left(\mathrm{NO}_{3}\right)(3.162(3) \AA),{ }^{11 \mathrm{a}}$ and $\left[\mathrm{Pd}_{2}(\mathrm{dppm})_{2}{ }^{-}\right.$ $\left.(\mathrm{CN})_{4}\right](3.276(1) \AA)^{21}$ The intermolecular Pd-Pd contact of $3.354(4) \AA$ in $\left[\mathrm{Pd}(\mathrm{bpy})(\mathrm{CN})_{2}\right]^{22}$ and the $\mathrm{Pt}-\mathrm{Pt}$ distance of $3.270(1) \AA$ in $\left[\mathrm{Pt}_{2}\left(\mathrm{~L}^{1}\right)_{2}(\mu\right.$-dppm $\left.)\right]\left(\mathrm{ClO}_{4}\right)_{2}{ }^{2}$ should also be noted. Since the latter is indicative of weak intermetal communication and the ionic radius of $\mathrm{Pt}(\mathrm{II})$ is larger than that for $\mathrm{Pd}(\mathrm{II})$, comparable intramolecular metal-metal separations in $\mathbf{1 e}, \mathbf{2 e}$, and $\left[\mathrm{Pt}_{2}\left(\mathrm{~L}^{1}\right)_{2}(\mu \text {-dppm) }]^{2+}\right.$ imply that the degree of ground-state metal-metal interaction is even smaller in the Pd system.

The small dihedral angles between the two $\left[\operatorname{Pd}\left(\mathrm{L}^{n}\right)\right]$ mean planes in $1 \mathbf{e}$ and $2 \mathrm{e}$ (5.7 and $4.8^{\circ}$ respectively) demonstrate that the intramolecular $\left[\mathrm{Pd}\left(\mathrm{L}^{n}\right)\right]$ units are nearly coplanar. The average interplanar separation between the $\mathrm{L}^{n}$ ligands in $\mathbf{1 e}$ and 2e are 3.34 and $3.35 \AA$, respectively. This feature and the small

(19) Yap, G. P. A.; Jensen, C. M. Inorg. Chem. 1992, 31, 4823.

(20) García-Herbosa, G.; Muñoz, A.; Miguel, D.; García-Granda, S. Organometallics 1994, 13, 1775.

(21) Yip, H. K.; Lai, T. F.; Che, C. M. J. Chem. Soc., Dalton Trans. 1991, 1639.

(22) Che, C. M.; He, L. Y.; Poon, C. K.; Mak, T. C. W. Inorg. Chem. 1989, 28,3081 . dihedral angle signify $\pi$-stacking interactions within the binuclear complexes, even though there is apparently minimal $\mathrm{Pd}-\mathrm{Pd}$ communication in the ground state. The torsion angle of $25.8^{\circ}$ (defined by $\mathrm{N}(2)-\mathrm{Pd}(1)-\mathrm{Pd}(2)-\mathrm{N}(4)$ ) between the two $\left[\mathrm{Pd}\left(\mathrm{L}^{1}\right)\right]$ moieties in $\mathbf{1 e}$ is smaller than the corresponding angle of $44.6^{\circ}$ in $\left[\mathrm{Pt}_{2}\left(\mathrm{~L}^{1}\right)_{2}(\mu\right.$-dppm) $]\left(\mathrm{ClO}_{4}\right)_{2} .^{1,2}$ The magnitude of the latter was attributed to optimized $\pi-\pi$ interactions between the aromatic ligands $\mathrm{L}^{1}$, to avoid repulsive forces arising from eclipsed overlap of $\pi$ orbitals (i.e. torsion angle ca. $0^{\circ}$ ). ${ }^{23}$ Hence it appears that, like the metal - metal interaction, the $\pi-\pi$ orbital overlap in $\mathbf{1 e}$ is inferior compared to the platinum analogue. The torsion angle between the two $\left[\mathrm{Pd}\left(\mathrm{L}^{2}\right)\right]$ moieties in $\mathbf{2 e}$ is $5.4^{\circ}$ (defined by $\left.\mathrm{N}(2)-\mathrm{Pd}(1)-\mathrm{Pd}(2)-\mathrm{N}(4)\right)$. There are no close intermolecular contacts between the aromatic ligands in the crystal lattices of $\mathbf{1 e}$ and $\mathbf{2 e}$ (distance $>4 \AA$ ).

Absorption Spectra. The UV-visible absorption spectra of the mono- and binuclear Pd(II) complexes depicted in this work have been obtained. The UV-vis spectral data of $\mathbf{1 a - 5 a}$ and $\mathbf{1 b}-\mathbf{1 d}$ in dichloromethane are listed in Table 3. For example, the absorption spectrum of 1a is shown in Figure 5. The intense absorptions at $\lambda_{\max }=272,278,309$, and $326 \mathrm{~nm}\left(\epsilon \sim 10^{4} \mathrm{dm}^{3}\right.$ $\mathrm{mol}^{-1} \mathrm{~cm}^{-1}$ ) are assigned to intraligand transitions of the cyclometalated ligand, based on similarities with the absorptions of the free ligand $\mathrm{HL}^{1}$. There is a moderately intense low-energy band tailing from $\lambda_{\max }=360-450 \mathrm{~nm}$ with a shoulder at $\lambda \sim$ $390 \mathrm{~nm}$. The $\epsilon$ value at $390 \mathrm{~nm}$ is $1150 \mathrm{dm}^{3} \mathrm{~mol}^{-1} \mathrm{~cm}^{-1}$ and we tentatively assign this band to a spin-allowed $4 \mathrm{~d}(\mathrm{Pd}) \rightarrow$ $\pi^{*}\left(\mathrm{~L}^{1}\right) \quad\left({ }^{1} \mathrm{MLCT}\right)$ transition. Absorption bands at $\lambda_{\max }=$ $349-400 \mathrm{~nm}$ in the related complexes $\left[\mathrm{Pd}(\mathrm{C}, \mathrm{N})_{2}\right](\mathrm{HC}, \mathrm{N}=$ 2-phenylpyridine, 2-(2'-thienyl)pyridine, 7,8-benzoquinoline) have also been ascribed to MLCT transitions, ${ }^{5}$ and the red shift of similar bands in analogous $\mathrm{Pt}(\mathrm{II})$ derivatives provides support for this assignment (see below). The MLCT transition of the cationic derivative $[\mathrm{Pd}($ terpy $) \mathrm{Cl}]\left[\mathrm{PF}_{6}\right]$ was previously assigned at $\lambda_{\max }=328-362 \mathrm{~nm} .{ }^{24}$ Ligand-field $\mathrm{d}-\mathrm{d}$ transitions have been invoked for absorptions in the $380-400 \mathrm{~nm}$ range for orthometalated azobenzene $\mathrm{Pd}(\mathrm{II})$ complexes, ${ }^{25}$ but unreasonably high $\epsilon$ values $\left(\right.$ ca. $8 \times 10^{3} \mathrm{dm}^{3} \mathrm{~mol}^{-1} \mathrm{~cm}^{-1}$ ) are reported. We reason that the $\epsilon$ value at $390 \mathrm{~nm}$ for $\mathbf{1 a}$ is also larger than would be expected for $\mathrm{d}-\mathrm{d}$ transitions in $\mathrm{Pd}(\mathrm{II})$ derivatives. Similar assignments can be made for complexes $\mathbf{1 b}, \mathbf{1 c}$, and $\mathbf{2 a}-\mathbf{5 a}$ : the absorptions at $\lambda_{\max } \leq 370 \mathrm{~nm}$ are assigned as intraligand in nature while the low-energy bands at $\lambda_{\max } \geq 390$ $\mathrm{nm}$ are attributed to MLCT transitions. The low-energy absorption for $\mathbf{1 d}$ is blue-shifted to $\lambda_{\max } 375 \mathrm{~nm}\left(\epsilon=1950 \mathrm{dm}^{3} \mathrm{~mol}^{-1}\right.$ $\mathrm{cm}^{-1}$ ) in $\mathrm{CH}_{3} \mathrm{CN}$, and this is consistent with the expected increase in the energy of the MLCT transition for cationic species.

The UV-visible spectral data of complexes $\mathbf{1 e - 5 e}$ and $\mathbf{1 f}$ in acetonitrile are listed in Table 4. The absorption spectrum of $\left[\mathrm{Pd}_{2}\left(\mathrm{~L}^{1}\right)_{2}(\mu \text {-dppm })\right]^{2+}(\mathbf{1 e})$ shows intense intraligand transitions at $\lambda_{\max } 309$ and $331 \mathrm{~nm}\left(\epsilon \sim 10^{4} \mathrm{dm}^{3} \mathrm{~mol}^{-1} \mathrm{~cm}^{-1}\right)$ and moderately intense and broad absorptions at $\lambda_{\max } 378-401 \mathrm{~nm}$ $\left(\epsilon=4900-3400 \mathrm{dm}^{3} \mathrm{~mol}^{-1} \mathrm{~cm}^{-1}\right)$ (Figure 6). All the absorption bands obey Beer's law in the concentration range $5 \times 10^{-6}$ to $5 \times 10^{-3} \mathrm{~mol} \mathrm{dm}^{-3}$. By comparison with the mononuclear counterpart 1d, it is evident that the spectra are very similar, except that the $\epsilon$ values are greater for 1e (Figure 6). This may be rationalized by an increase in the number of $\operatorname{Pd}\left(\mathrm{L}^{1}\right)$ chromophores per molecule. For $\mathrm{d}^{8}$ systems, a $\mathrm{d} \sigma^{*} \rightarrow \pi^{*}$ metal-

(23) Hunter, C. A.; Sanders, J. K. M. J. Am. Chem. Soc. 1990, 112, 5525.

(24) Zhang, W.; Bensimon, C.; Crutchley, R. J. Inorg. Chem. 1993, 32, 5808.

(25) Wakatsuki, Y.; Yamazaki, H.; Grutsch, P. A.; Santhanam, M.; Kutal, C. J. Am. Chem. Soc. 1985, 107, 8153 . 
Table 3. UV-Visible Spectral Data of Mononuclear Complexes ${ }^{a}$

\begin{tabular}{lc}
\hline \multicolumn{1}{c}{ complex } & $\lambda_{\max } / \mathrm{nm}_{\left(\epsilon / \mathrm{dm}^{3} \mathrm{~mol}^{-1} \mathrm{~cm}^{-1}\right)}$ \\
\hline $\mathrm{Pd}\left(\mathrm{L}^{1}\right) \mathrm{Cl}, \mathbf{1 a}$ & $272(27300), 278(27600), 309(15900), 326(15900), 342(\mathrm{sh}, 9500), 390(\mathrm{sh}, 1150), 421(\mathrm{sh}, 350)$ \\
$\mathrm{Pd}\left(\mathrm{L}^{2}\right) \mathrm{Cl}, \mathbf{2 a}$ & $263(26700), 287(40600), 328(18600), 349(\mathrm{sh}, 11500), 394(\mathrm{sh}, 1600), 422(\mathrm{sh}, 510)$ \\
$\mathrm{Pd}\left(\mathrm{L}^{3}\right) \mathrm{Cl}, \mathbf{3 a}$ & $266(25400), 291(39900), 329(18600), 350(\mathrm{sh}, 12000), 401(\mathrm{sh}, 1350), 428(\mathrm{sh}, 370)$ \\
$\mathrm{Pd}\left(\mathrm{L}^{4}\right) \mathrm{Cl}, \mathbf{4 a}$ & $263(23000), 294(33400), 329(18 \mathrm{a} 00), 346(\mathrm{sh}, 13000), 369(\mathrm{sh}, 5100), 397(\mathrm{sh}, 1500), 422(450)$ \\
$\mathrm{Pd}\left(\mathrm{L}^{5}\right) \mathrm{Cl}, \mathbf{5 a}$ & $262(26700), 278(26300), 312(34500), 335(\mathrm{sh}, 25100), 347(\mathrm{sh}, 20100), 369(\mathrm{sh}, 11400), 398$ \\
& $(\mathrm{sh}, 2350), 423(540)$ \\
$\mathrm{Pd}\left(\mathrm{L}^{1}\right) \mathrm{Br}, \mathbf{1 b}$ & $272(22300), 279(23000), 311(15300), 326(14700), 343(\mathrm{sh}, 9000), 395(\mathrm{sh}, 1100), 418(\mathrm{sh}, 410)$ \\
$\mathrm{Pd}\left(\mathrm{L}^{1}\right) \mathrm{I}, \mathbf{1 c}$ & $249(28500), 268(22000), 317(15800), 328(15000), 390(\mathrm{sh}, 2200), 418(\mathrm{sh}, 940)$ \\
{$\left[\mathrm{Pd}\left(\mathrm{L}^{1}\right) \mathrm{PPh}_{3}\right]\left(\mathrm{ClO}_{4}\right), \mathbf{1 d}\left(\mathrm{ClO}_{4}\right)^{b}$} & $243(48300), 267(\mathrm{sh}, 26400), 306(13000), 331(\mathrm{sh}, 10400), 375(1950), 397(\mathrm{sh}, 1250)$ \\
${ }^{a} \mathrm{In} \mathrm{CH}_{2} \mathrm{Cl}_{2}$ unless stated otherwise. ${ }^{b}$ In acetonitrile.
\end{tabular}

Table 4. UV-Visible Spectral Data of Binuclear Complexes in Acetonitrile

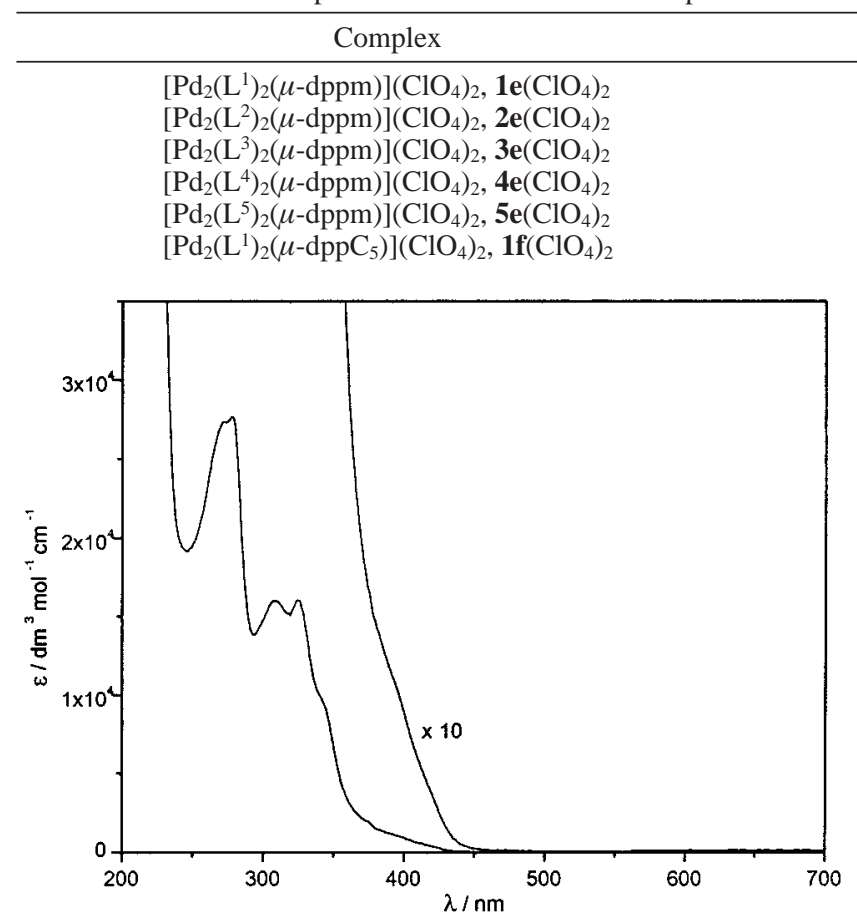

Figure 5. UV-vis absorption spectrum of $1 \mathbf{a}$ in $\mathrm{CH}_{2} \mathrm{Cl}_{2}$ at $298 \mathrm{~K}$.

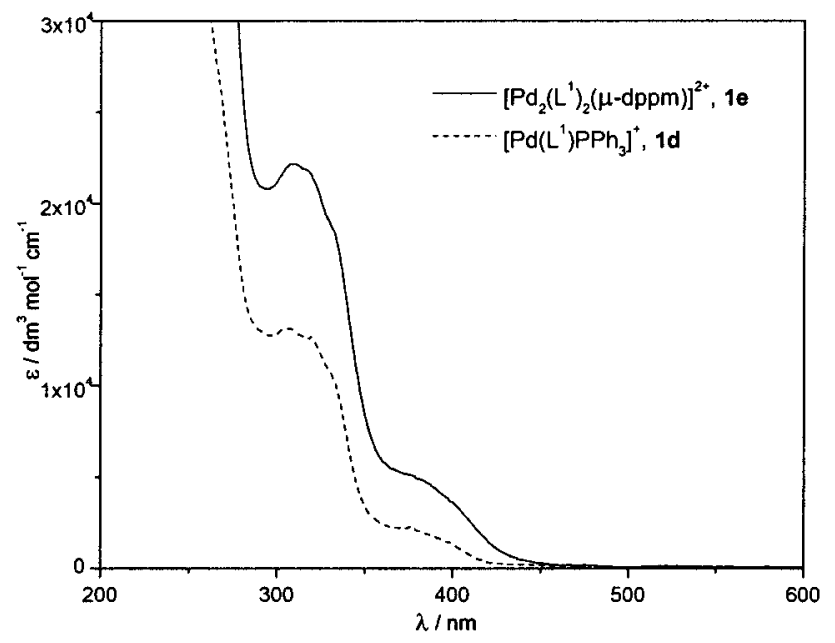

Figure 6. $\mathrm{UV}-$ vis absorption spectra of $1 \mathrm{~d}$ and $1 \mathrm{e}$ in $\mathrm{CH}_{3} \mathrm{CN}$ at $298 \mathrm{~K}$.

metal-to-ligand charge transfer (MMLCT) transition which is red-shifted from the MLCT transition of mononuclear species is expected for binuclear derivatives exhibiting significant metal-metal interaction (Figure 7). ${ }^{1,26}$ The virtual match

(26) Miskowski, V. M.; Houlding, V. H. Inorg. Chem. 1991, 30, 4446. $\lambda_{\max } / \mathrm{nm}\left(\epsilon / \mathrm{dm}^{3} \mathrm{~mol}^{-1} \mathrm{~cm}^{-1}\right)$

309 (22 000), 331 (sh, 18 500), 378 (4900), 401 (sh, 3400)

286 (69 800), 311 (sh, 44 400), 386 (4900), 408 (sh, 3200)

288 (71 300), 314 (sh, 51 100), 390 (4800), 412 (sh, 3100)

291 (57 400), 316 (49 500), 387 (4700), 410 (sh, 2700)

303 (46 200), 330 (51 300), 396 (sh, 7100), 418 (sh, 2900)

307 (25 600), 318 (25 100), 378 (4700), 398 (sh, 3100)

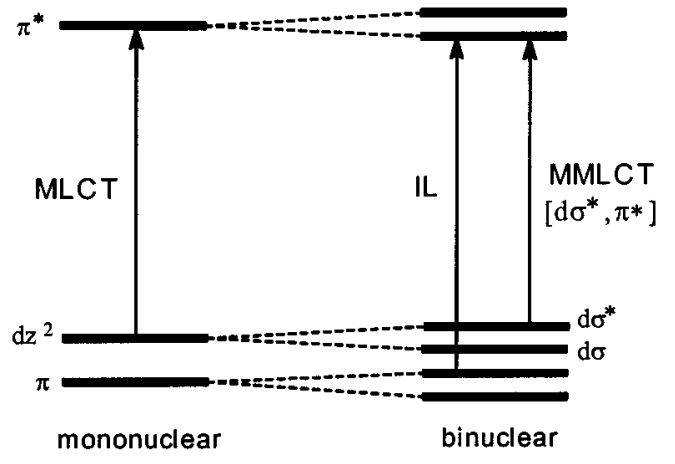

Figure 7. Schematic molecular orbital diagram illustrating metalmetal and ligand-ligand interactions in binuclear polypyridine $\mathrm{d}^{8}-\mathrm{d}^{8}$ complexes.

between the low-energy absorptions at $\lambda>360 \mathrm{~nm}$ for $\mathbf{1 d}$ and 1e, together with the structural parameters (see above), thus imply very weak or minimal $\mathrm{Pd}-\mathrm{Pd}$ interaction in 1e. Accordingly, the absorptions at $\lambda_{\max }=378-401 \mathrm{~nm}$ for $1 \mathbf{e}$ are tentatively assigned as MLCT in nature like for 1d. Furthermore, the low-energy absorption of $\mathbf{1 f}$, in which $\left[\mathrm{Pd}\left(\mathrm{L}^{1}\right)\right]$ units are separated by a long diphosphine ligand and $\mathrm{Pd}-\mathrm{Pd}$ communication is disfavored, is almost identical to that for 1e (Table 4). This observation reinforces the notion that there is minimal ground-state metal-metal interaction in these binuclear Pd(II) complexes. Similar assignments can be made with respect to the absorption spectra of $\mathbf{2 e - 5 e}$.

Emission Spectra. All complexes in this account are nonemissive at room temperature. At $77 \mathrm{~K}$, the cationic derivatives bearing phosphine ligands generally display more intense emissions than neutral species (Table 5). Indeed, the $\left[\mathrm{Pd}\left(\mathrm{L}^{1-5}\right) \mathrm{Cl}\right]$ $(\mathbf{1 a}-\mathbf{5 a})$ and $\left[\mathrm{Pd}\left(\mathrm{L}^{1}\right) \mathrm{X}\right](\mathrm{X}=\mathrm{Br}, \mathbf{1 b} ; \mathrm{I}, \mathbf{1 c})$ solids are nonemissive at 298 and $77 \mathrm{~K}^{27}$ The cationic complex $\left[\mathrm{Pd}\left(\mathrm{L}^{1}\right)\left(\mathrm{PPh}_{3}\right)\right]^{+}$ (1d) and the related dication $\left[\mathrm{Pd}_{2}\left(\mathrm{~L}^{1}\right)_{2}\left(\mu \text {-dppC } \mathrm{C}_{5}\right)\right]^{2+}$ (1f) display vibronically structured emissions at $\lambda_{\max }=467-576$ and 474-586 nm, respectively, in $77 \mathrm{~K}$ glassy $\mathrm{MeOH} / \mathrm{EtOH}$ solutions, with vibronic progressions of ca. $1300 \mathrm{~cm}^{-1}$ (Figure 8 ) and lifetimes $(\tau)$ in the order of $10^{-4} \mathrm{~s}$. These highenergy bands can be assigned to intraligand ( $\left.{ }^{3} \mathrm{IL}\right)$ emission by the cyclometalated ligand $\mathrm{L}^{1}$. The cationic derivatives $\mathbf{1 e}-$

(27) These complexes are weakly emissive in frozen dichloromethane at $77 \mathrm{~K}$. 
Table 5. $77 \mathrm{~K}$ Emission Data (complex concentration $1 \times 10^{-4} \mathrm{M}$ )

\begin{tabular}{cll}
\hline & \multicolumn{2}{c}{$\lambda_{\max } / \mathrm{nm}$} \\
\cline { 2 - 3 } complex & \multicolumn{1}{c}{ MeOH/EtOH $(1: 1)$} & Solid \\
\hline$\left[\mathrm{Pd}_{2}\left(\mathrm{~L}^{1}\right)_{2}(\mu\right.$-dppm $\left.)\right]\left(\mathrm{ClO}_{4}\right)_{2}, \mathbf{1 e}\left(\mathrm{ClO}_{4}\right)_{2}$ & $480,510,626$ & 526,553 \\
{$\left[\mathrm{Pd}_{2}\left(\mathrm{~L}^{2}\right)_{2}(\mu\right.$-dppm $\left.)\right]\left(\mathrm{ClO}_{4}\right)_{2}, \mathbf{2 e}\left(\mathrm{ClO}_{4}\right)_{2}$} & $480,523,633$ & $516(\max ), 556$ \\
{$\left[\mathrm{Pd}_{2}\left(\mathrm{~L}^{3}\right)_{2}(\mu\right.$-dppm $\left.)\right]\left(\mathrm{ClO}_{4}\right)_{2}, \mathbf{3 e}\left(\mathrm{ClO}_{4}\right)_{2}$} & $480,523,658$ & 529,566 \\
{$\left[\mathrm{Pd}_{2}\left(\mathrm{~L}^{4}\right)_{2}(\mu\right.$-dppm $\left.)\right]\left(\mathrm{ClO}_{4}\right)_{2}, \mathbf{4 e}\left(\mathrm{ClO}_{4}\right)_{2}$} & $478,514,632$ & $544(\max ), 578$ \\
{$\left[\mathrm{Pd}_{2}\left(\mathrm{~L}^{5}\right)_{2}(\mu\right.$-dppm) $]\left(\mathrm{ClO}_{4}\right)_{2}, \mathbf{5 e}\left(\mathrm{ClO}_{4}\right)_{2}$} & $479(\mathrm{sh}), 517,634$ & $526(\max ), 560$ \\
{$\left[\mathrm{Pd}_{2}\left(\mathrm{~L}^{1}\right)_{2}(\mu\right.$-dppC } & \\
{$\left[\mathrm{Pd}\left(\mathrm{L}^{1}\right) \mathrm{PPh}_{3}\right]\left(\mathrm{ClO}_{4}\right), \mathbf{1 d}\left(\mathrm{ClO}_{4}\right)_{2}, \mathbf{1 f}\left(\mathrm{ClO}_{4}\right)_{2}$} & $474(\max ), 507,542,586(\mathrm{sh})$ & $498(\max ), 527$ \\
& $467(\max ), 501,532,576(\mathrm{sh})$ & $493(\max ), 527,563$
\end{tabular}

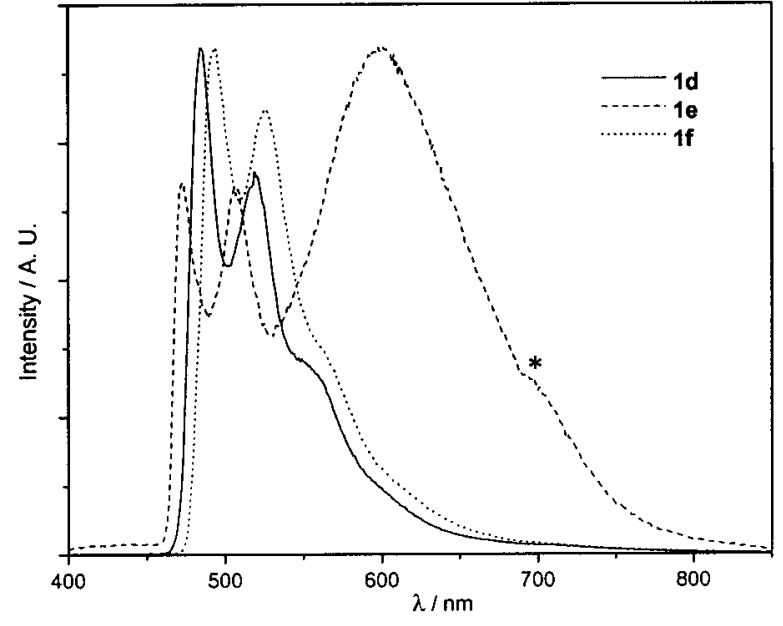

Figure 8. Normalized $77 \mathrm{~K}$ emission spectra of $\mathbf{1 d}, \mathbf{1 e}$, and $\mathbf{1 f}$ in $1: 1 \mathrm{MeOH} / \mathrm{EtOH}$ glass $\left(\lambda_{\mathrm{ex}}=350 \mathrm{~nm}, *\right.$ denotes instrumental artifact).

$\mathbf{5 e}\left(\mathrm{ClO}_{4}\right)_{2}, \mathbf{1 d}\left(\mathrm{ClO}_{4}\right)$, and $\mathbf{1 f}\left(\mathrm{ClO}_{4}\right)_{2}$ are luminescent in the solid state at $77 \mathrm{~K} .{ }^{28}$ Except for $\mathbf{1 e}\left(\mathrm{ClO}_{4}\right)_{2}$ and $\mathbf{3 e}\left(\mathrm{ClO}_{4}\right)_{2}$, highly structured emissions at $\lambda_{\max }=493-578 \mathrm{~nm}$ are displayed, and these are ascribed to metal-perturbed ${ }^{3} \mathrm{IL}$ excited states. The solid emissions of $1 \mathbf{e}\left(\mathrm{ClO}_{4}\right)_{2}\left(\lambda_{\max }=526-553 \mathrm{~nm}\right)$ and $3 \mathbf{e}\left(\mathrm{ClO}_{4}\right)_{2}\left(\lambda_{\max }=529-566 \mathrm{~nm}\right)$ are more diffuse.

Complexes $\mathbf{1 e}-\mathbf{5 e}$ are luminescent in glassy $\mathrm{MeOH} / \mathrm{EtOH}$ solutions (Table 5). The emission spectrum of 1e (Figure 8) shows sharp bands at $\lambda_{\max }=480$ and $510 \mathrm{~nm}(\tau=160 \mu \mathrm{s}$, vibronic progression $1225 \mathrm{~cm}^{-1}$ ) and a broad structureless band at $626 \mathrm{~nm}(\tau=90 \mu \mathrm{s})$. The high-energy structure resembles the intraligand emission of $\mathbf{1 d}$ and $\mathbf{1 f}$ and is therefore assigned to a ${ }^{3} \mathrm{IL}$ excited state. The broad emission at $626 \mathrm{~nm}$ is tentatively assigned to an excimeric ${ }^{3} \mathrm{IL}$ transition resulting from $\pi-\pi$ interactions. ${ }^{29}$ There are evidently various static conformations for 1e in glassy alcohol solution, and only some of these can exhibit substantial excimeric character in their lowest ${ }^{3} \mathrm{IL}$ excited states. A d $\sigma^{*} \rightarrow \pi^{*}$ MMLCT assignment is not preferred due to the lack of evidence for appreciable $\mathrm{Pd}-\mathrm{Pd}$ bonding. The absence of a low-energy band for $\mathbf{1 d}$ and $\mathbf{1 f}$ suggests that their geometry disfavor such $\pi-\pi$ interactions. Complexes $2 \mathbf{e}$ and 5e exhibit dual emissions at $\lambda_{\max }=480,523(\tau=64 \mu \mathrm{s})$, and $633 \mathrm{~nm}(\tau=88 \mu \mathrm{s})$ and $\lambda_{\max }=479(\mathrm{sh}), 517(\tau=74 \mu \mathrm{s})$, and $634 \mathrm{~nm}(\tau=16 \mu \mathrm{s})$, respectively, in MeOH/EtOH glasses. Like the $\mathrm{Pt}(\mathrm{II})$ analogues, ${ }^{1}$ no apparent photophysical trend arising from the various 4-aryl substituents is observed.

Our investigation has shown that the nature of the luminescent excited states of $\mathbf{1 e}-\mathbf{5 e}$ is highly dependent upon the solvent and medium. For example, their emission spectra in frozen acetonitrile at $77 \mathrm{~K}$ contain structureless bands at $\lambda_{\max } \sim 600$

(28) Non-linear exponential decays for solid-state emission lifetimes were observed.

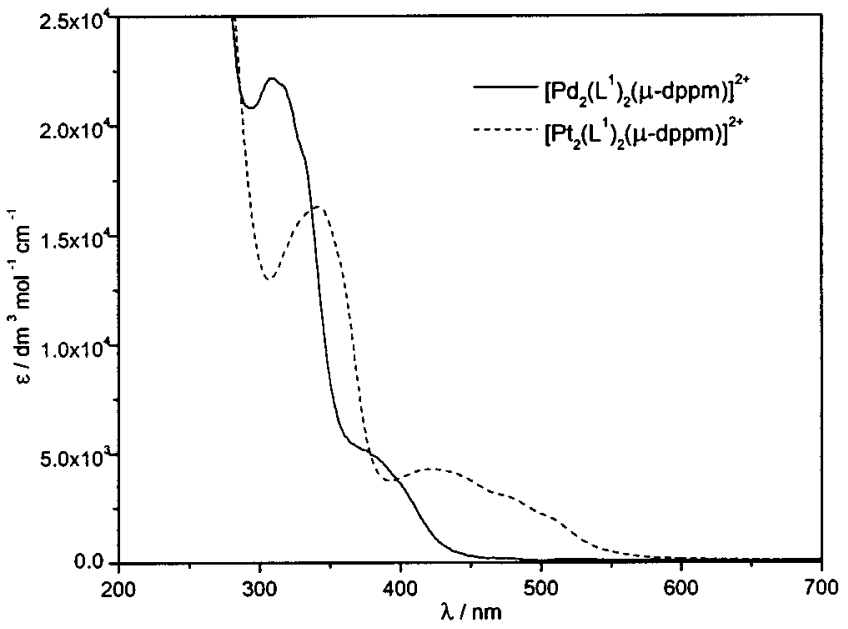

Figure 9. UV-vis absorption spectra of $\left[\mathrm{M}_{2}\left(\mathrm{~L}^{1}\right)_{2}(\mu \text {-dppm })\right]^{2+}(\mathrm{M}=$ $\mathrm{Pd}, \mathrm{Pt}$ ) in $\mathrm{CH}_{3} \mathrm{CN}$ at $298 \mathrm{~K}$.

nm only (assignable to $\pi-\pi$ excimeric ${ }^{3} \mathrm{IL}$ transitions), and no high-energy vibronic bands are detected as in $\mathrm{MeOH} / \mathrm{EtOH}$ glasses. Changes in emissive excited states in different solvents have been reported for binuclear $\mathrm{Pt}(\mathrm{II})$ terpyridine complexes. ${ }^{30}$ The $\pi$-stacking conformations leading to an excimeric lowenergy emission are apparently facilitated in rigid, frozen solutions; $\mathbf{1 e}-\mathbf{5 e}\left(\mathrm{ClO}_{4}\right)_{2}$ solids do not emit at $\lambda_{\max } \geq 600 \mathrm{~nm}$. This is consistent with the X-ray structural data that revealed non-ideal $\pi-\pi$ interactions in the crystal lattices. The structured solid-state emissions (Table 5) are slightly red-shifted from those of $\mathrm{MeOH} / \mathrm{EtOH}$ glasses, perhaps due to very weak $\pi-\pi$ interactions.

Comparisons between $\operatorname{Pd}($ II) and Pt(II) Systems. A principal objective of this work is to highlight spectroscopic similarities and differences between the cyclometalated 6phenyl-2,2'-bipyridine Pd(II) and Pt(II) congeners, ${ }^{1,2}$ in order to shed further light upon the nature of their excited states and intramolecular $\left(\mathrm{d}^{8}-\mathrm{d}^{8}\right.$ and $\left.\pi-\pi\right)$ interactions.

The absorption at $\lambda \sim 390 \mathrm{~nm}$ for $\left[\mathrm{Pd}\left(\mathrm{L}^{1}\right) \mathrm{Cl}\right](\mathbf{1 a})$ in $\mathrm{CH}_{2} \mathrm{Cl}_{2}$ is assigned to a MLCT transition. Since the $4 \mathrm{~d}(\mathrm{Pd})$ orbital exhibits a higher ionization potential than $5 \mathrm{~d}(\mathrm{Pt})$, a red shift for the corresponding absorption in $\left[\mathrm{Pt}\left(\mathrm{L}^{1}\right) \mathrm{Cl}\right]\left(\lambda_{\max }=430 \mathrm{~nm}\right.$ in $\mathrm{CH}_{2} \mathrm{Cl}_{2}$ ) is consistent with this assignment. Shifting of MLCT transitions to lower energies for $[\mathrm{M}(\mathrm{bph})(\mathrm{bpy})]\left(\mathrm{H}_{2} \mathrm{bph}=\right.$ biphenyl) from $\mathrm{Pd}(\mathrm{II})$ to $\mathrm{Pt}(\mathrm{II})$ has been reported. ${ }^{31}$ A compari-

(29) Comparable emissions have been observed for Pt(II) terpyridine complexes: (a) Bailey, J. A.; Hill, M. G.; Marsh, R. E.; Miskowski, V. M.; Schaefer, W. P.; Gray, H. B. Inorg. Chem. 1995, 34, 4591. (b) Arena, G.; Calogero, G.; Campagna, S.; Scolaro, L. M.; Ricevuto, V.; Romeo, R. Inorg. Chem. 1998, 37, 2763. (c) Lai, S. W.; Chan, M. C. W.; Cheung, K. K.; Che, C. M. Inorg. Chem. 1999, 38, 4262.

(30) Bailey, J. A.; Miskowski, V. M.; Gray, H. B. Inorg. Chem. 1993, 32 , 369.

(31) Cornioley-Deuschel, C.; von Zelewsky, A. Inorg. Chem. 1987, 26, 3354. 


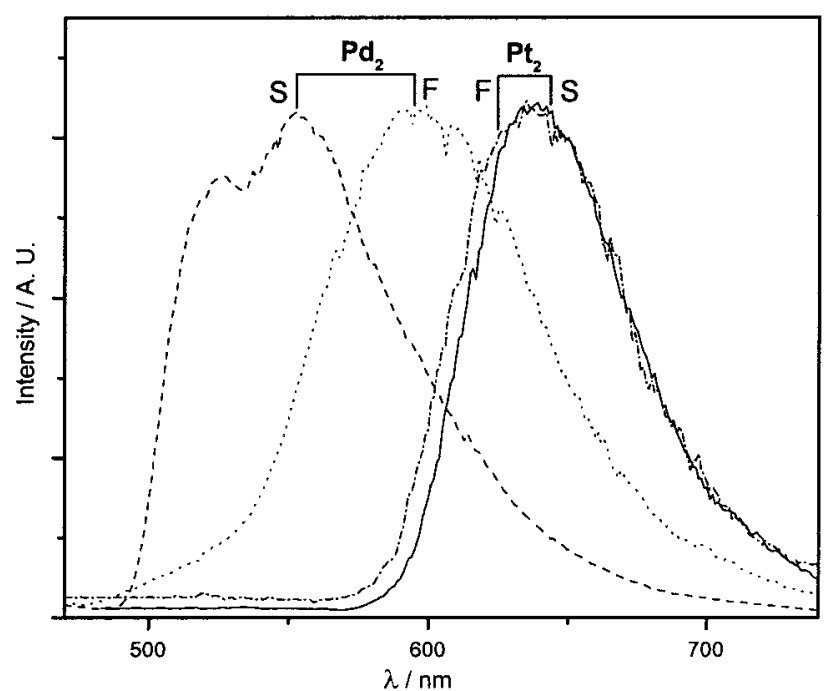

Figure 10. Normalized $77 \mathrm{~K}$ emission spectra $\left(\lambda_{\mathrm{ex}}=350 \mathrm{~nm}\right)$ of $\left[\mathrm{M}_{2}\left(\mathrm{~L}^{1}\right)_{2}(\mu\right.$-dppm $\left.)\right]\left(\mathrm{ClO}_{4}\right)_{2}(\mathrm{M}=\mathrm{Pd}, \mathrm{Pt})$ in frozen $\mathrm{CH}_{3} \mathrm{CN}(\mathrm{F})$ and solid state $(\mathrm{S})$.

son between the UV absorption spectra of $\left[\mathrm{Pd}_{2}\left(\mathrm{~L}^{1}\right)_{2}(\mu \text {-dppm) }]^{2+}\right.$ (1e) and its $\mathrm{Pt}(\mathrm{II})$ analogue is depicted in Figure 9. The platinum derivative has noticeably higher absorbance at $\lambda_{\max }=450-$ $600 \mathrm{~nm}$, a region where mononuclear counterparts do not absorb, and a $\mathrm{d} \sigma^{*} \rightarrow \pi^{*}$ transition was previously assigned. We again conclude that the apparent blue shift in the lowest energy band for 1e signifies exceedingly weak/minimal $\mathrm{Pd}-\mathrm{Pd}$ interaction in the ground state.
Correlation of luminescent properties between the $\mathrm{Pd}(\mathrm{II})$ and $\mathrm{Pt}(\mathrm{II})$ counterparts is also informative. The $77 \mathrm{~K}$ emission of $\left[\mathrm{M}_{2}\left(\mathrm{~L}^{1}\right)_{2}(\mu \text {-dppm })\right]^{2+}$ in frozen $\mathrm{CH}_{3} \mathrm{CN}$ occurs at $\lambda_{\max }=598$ and $638 \mathrm{~nm}$ for $\mathrm{Pd}$ and $\mathrm{Pt}$, respectively (Figure 10). Since a $\pi-\pi$ excimeric ${ }^{3} \mathrm{IL}$ transition is invoked for the $\mathrm{Pd}_{2}$ species, the red shift for the $\mathrm{Pt}_{2}$ analogue is indicative of an excitedstate arising from $\pi-\pi$ (excimeric ${ }^{3} \mathrm{IL}$ ) and metal-metal (previously assigned as $\mathrm{d} \sigma^{*} \rightarrow \pi^{*}$ ) interactions. In other words, the $\mathrm{Pd}-\mathrm{Pd}$ interaction in the excited state appears weaker. The $77 \mathrm{~K}$ solid-state emission spectra for $\left[\mathrm{M}_{2}\left(\mathrm{~L}^{1}\right)_{2}(\mu\right.$-dppm $\left.)\right]\left(\mathrm{ClO}_{4}\right)_{2}$ illustrate a similar trend (Figure 10). A structureless band appears at $\lambda_{\max }=640 \mathrm{~nm}$ for $\mathrm{Pt}_{2}$, while a structured emission is observed at $\lambda_{\max }=526$ and $553 \mathrm{~nm}$ for $\mathrm{Pd}_{2}$. The nature of the former is again ascribed to mixed $\mathrm{d} \sigma^{*} \rightarrow \pi^{*}$ /excimeric $\pi$ $\rightarrow \pi^{*}$, while the excited state of the $\operatorname{Pd}_{2}$ species is assigned as ${ }^{3} \mathrm{IL}$ accompanied by weak $\pi-\pi$ excimeric character.

Acknowledgment. We are grateful for financial support from The University of Hong Kong and the Research Grants Council of the Hong Kong SAR, China [HKU 7298/99P]. We thank Dr. V. M. Miskowski for helpful discussions.

Supporting Information Available: Listings of crystal data, atomic coordinates, calculated coordinates, anisotropic displacement parameters, and bond lengths and angles for $\mathbf{1} \mathbf{d}\left(\mathrm{ClO}_{4}\right), \mathbf{1}\left(\mathrm{ClO}_{4}\right)_{2} \cdot \mathrm{DMF}$, and $2 \mathbf{e}\left(\mathrm{ClO}_{4}\right)_{2}$. This material is available free of charge via the Internet at http://pubs.acs.org.

IC991089G 